\title{
MORE ON CARDINAL ARITHMETIC SH410
}

\author{
SAHARON SHELAH \\ Institute of Mathematics \\ The Hebrew University \\ Jerusalem, Israel \\ Rutgers University \\ Mathematics Department \\ New Brunswick, NJ USA
}

Done 1990

Partially supported by the Basic Research Fund, Israel Academy of Science

I thank Alice Leonhardt for the beautiful typing

Publ. 410

Latest Revision - 02/March/12 


\section{An Annotated Table of Contents}

\section{$\S 1 \quad$ On Normal Filters}

In $[$ Sh 355, §4] and [Sh 400, §3,§5] we have computed $\operatorname{cov}(\lambda, \lambda, \theta, \sigma)$ when $\theta>\sigma=$ cf $\sigma>\aleph_{0}$, using tcf $\prod_{i<\kappa} \lambda_{i} / J$, for $\sigma$-complete ideals $J$ and $\sigma \leq \kappa<\theta$. In [Sh 371, $\left.§ 4\right]$ we deal with a similar theorem where we restrict ourselves to normal ideals, namely prc, but its computation, using pp's, did not always yield exact values (i.e. the upper and lower bounds tend not to match). Here we give reasonably exact values for $\operatorname{prc}_{J}(f, \bar{\mu})$, using the true cofinalities of $\prod_{i<\kappa} \mu_{i}^{\prime} / J_{1}$, where $J_{1}$ is a normal filter on $\kappa$ extending $J$ and for $i<\kappa, \mu_{i}^{\prime}$ is a regular ordinal satisfying $\mu_{i} \leq \mu_{i}^{\prime} \leq f(i)$. We also give a sufficient condition for the existence of normal ideal $J$ on $\kappa$ such that for some sequence $\left\langle\lambda_{i}: i<\kappa\right\rangle$ of regulars, we have $\lambda=\operatorname{tim}\left\langle\lambda_{i}: i<\kappa\right\rangle, \mu=\operatorname{tcf} \prod_{i<\kappa} \lambda_{i} / J$.

$\S 2$ On measures of the size of $[\lambda]^{<\kappa}$

We mainly investigate cardinals like

$$
\begin{aligned}
& \operatorname{Min}\left\{|\mathscr{P}|: \mathscr{P} \subseteq[\lambda]^{<\theta}(\lambda) \text { and for every } Z \in \mathscr{S}_{\leq \kappa}(\lambda)\right. \text { there is a sequence } \\
&\left\langle Z_{n}: n<\omega\right\rangle \text { of subsets of } Z \text { such that } Z=\bigcup_{n<\omega} Z_{n} \text { and } \\
&\left.(\forall n<\omega)\left(\forall y \in \mathscr{S}_{<\theta}\left(Z_{n}\right)\right)(\exists z \in \mathscr{P})[y \subseteq z]\right\} .
\end{aligned}
$$

We also give sufficient conditions for the strong covering to hold for a pair $(W, V)$ of universes.

$\S 3$ pcf - Inaccessibility and characterizing the existence of non $<{ }_{J}$-decreasing sequences (for topology)

We restate various results using pcf inaccessibility and present more consequences of the proofs in [Sh 400, $§ 2, \S 4]$. We characterize those $\kappa<\sigma<\theta$ for which there is a sequence $\left\langle f_{\alpha}: \alpha<\theta\right\rangle$ of members of ${ }^{\kappa} \sigma$ such that $\alpha<\beta \Rightarrow f_{\alpha} \not \leq f_{\beta}$; answering a question of Gerlits, Hajnal and Szentmiklossy. [See more in [Sh 513, §6]].

\section{$\S 4 \quad$ Entangled Orders - Narrow Order Boolean Algebras Revisited}

We show that for a class of cardinals $\lambda$ there is an entangled linear order of cardinality $\lambda^{+}$. This holds for $\lambda$ if there is a $\kappa$ such that $\kappa^{+4} \leq \operatorname{cf}(\lambda)<\lambda \leq 2^{\kappa}$. [See more in [Sh 462] and [Sh 666].]

$\S 5$ prd: Measuring $\prod_{i<\kappa} f(i)$ by a family of ideals and a family of sequences $\left\langle B_{i}: i<\kappa\right\rangle,\left|B_{i}\right|<\mu_{i}$

This generalizes Section 1, replacing normality by an abstract property; we also present a generalization of the concept of a normal filter, and deduce $\operatorname{prd}_{J}(\bar{f}, \bar{\mu}) \leq$ 
$\S 6 \quad$ The Existence of Strongly Almost Disjoint Families

We characterize such existence questions by pp's. An example is the question of the existence of a family of $\lambda^{+}$subsets of $\lambda>\kappa^{\aleph_{0}}$, each of cardinality $\kappa\left(>\aleph_{0}\right)$ such that the intersection of any two is finite. 


\section{$\S 1$ On Normal Filters}

The following Lemma 1.2 is similar to [Sh 355, 5.4], [Sh 400, 3.5], but deal with normal ideals (see [Sh 371, §4], in particular [\Sh:374], Definition 4.1, Claim ?). $\rightarrow \quad$ scite $\{4.6\}$ undefined

Remember prc is defined in [Sh 371, §4] as:

1.1 Definition. 1) For a regular uncountable cardinal $\kappa$, normal ideal $J$ on $\kappa, \bar{\mu}$ a $\kappa$-sequence of cardinals $>\kappa$, and $f \in{ }^{\kappa}$ Ord, we define:

$$
\begin{aligned}
\operatorname{prc}_{J}(f, \bar{\mu})=\operatorname{Min}\{|\mathscr{P}|: & \mathscr{P} \text { is a family of } \kappa \text {-sequences of sets of ordinals, } \\
& \bar{B}=\left\langle B_{i}: i<\kappa\right\rangle,\left|B_{i}\right|<\mu_{i} \text { or at least } \\
& \left\{i<\kappa:\left|B_{i}\right| \geqq \mu_{i}\right\} \in J, \text { such that: for every } g \in{ }^{\kappa} \text { Ord, } \\
& g \leqq J f \text { there is a sequence }\left\langle\bar{A}^{\zeta}: \zeta<\kappa\right\rangle \text { of members } \\
& \left.\mathscr{P} \text { satisfying }\left\{i<\kappa: g(i) \notin \bigcup \bigcup_{\zeta<i} A_{i}^{\zeta}\right\} \in J\right\} .
\end{aligned}
$$

2) We may write $f$ as a sequence of ordinals say $\left\langle\lambda_{i}: i<\kappa\right\rangle$, and if $\lambda_{i}=\lambda$ for each $i$, we may write $\lambda$.

3) $\operatorname{prc}_{J}^{\prime}(f, \mu)$ is defined similarly but from $\bar{B}=\left\langle B_{i}: i<\kappa\right\rangle$ we demand this time $\left|B_{i}\right|<\mu$.

Remark. See there ([Sh 371, 4.2,4.3]) for some basic properties. But 1.2 below substantially improves [Sh 371, Claim 4.6] there.

1.2 Lemma. 1) Let $\kappa$ be a regular uncountable cardinal, $f: \kappa \rightarrow$ ordinals, $J$ a normal ideal on $\kappa$, and $\bar{\mu}=\left\langle\mu_{i}: i<\kappa\right\rangle$ a sequence of regular cardinals. Then

$$
\begin{aligned}
\operatorname{prc}_{J}(f, \bar{\mu})=\sup \left\{\operatorname{tcf}\left[\prod_{i<\kappa} \mu_{i}^{\prime} / J_{1}\right]:\right. & J_{1} \text { a normal ideal on } \kappa \text { extending } J \\
& \text { such that the tcf is well defined and } \\
& \left.\left\{i<\kappa: \text { not " } \mu_{i} \leqq \mu_{i}^{\prime}=\operatorname{cf}\left(\mu_{i}^{\prime}\right) \leqq f(i) \text { " }\right\} \in J\right\}
\end{aligned}
$$

provided that:

( $\alpha) \mu_{i}=\mu>\kappa$.

2) We can replace assumption $(\alpha)$ by $(\beta)$ below, and $\bigwedge_{i} \mu_{i}>\kappa$.

( $\beta) \mu_{i}$ strictly increasing and for limit $i$ :

if $i=\sum \mu_{j}$ is regular then $i=\mu_{i}$, otherwise $\mu_{i}=\left(\sum \mu_{j}\right)^{+}$. 
1.3 Remark. 1) On getting $=^{+}$see $[$Sh 420, 6.1(C)] and [Sh 430, §4]. The problem is when $\operatorname{pcf}(\mathfrak{a})$ has an accumulation point which is inaccessible.

2) In the case $(\beta)$ holds, if $\bar{\mu}^{*}=\left\langle\mu_{i}^{*}: i<\kappa\right\rangle$ is (strictly) increasing continuous, $\sup _{i<\kappa} \mu_{i}^{*}=\sup _{i<\kappa} \mu_{i}$ then $\operatorname{prc}(f, \bar{\mu})+\left(\sum_{i<\kappa} \mu_{i}\right)^{+}=\operatorname{prc}\left(f, \bar{\mu}^{*}\right)+\left(\sum_{i<\kappa} \mu_{i}\right)^{+}$, by [Sh 371, $4.10(2)]+[$ Sh 355, 2.1].

3) If in $(\beta)$ we place " $\mu_{i}>\kappa$ " by $\sup _{i<\kappa} \mu_{i}=\kappa, \bigwedge_{i<\kappa^{*}} \mu_{i}<\kappa$ (so $\kappa$ is inaccessible we can get:

$$
\operatorname{prc}_{J}(f, \bar{\mu})=\sup \left\{\operatorname{nor}_{-c f} \prod_{i<\kappa} \mu_{i}^{\prime} / J:\left\{i: \mu_{i} \leq \mu_{i}^{\prime}=\operatorname{cf}\left(\mu_{i}^{\prime}\right) \leq f(i)\right\} \in J\right\} .
$$

Proof. The inequality $\geq$ :

Same proof as that of " $\lambda(1) \leq \lambda(2)$ " in the proof of $[$ Sh 371, 4.6].

The inequality $\leq$ :

Let $\lambda^{*}$ be the successor of the sup.

1.4 Fact. There is a family $\mathscr{P}^{*}$ such that:

(i) members of $\mathscr{P}^{*}$ are of the form

$$
\left\langle B_{i, \zeta}: i<\kappa, \zeta<\zeta_{i}\right\rangle \text { or }\left\langle<B_{i, \zeta}: \zeta<\zeta_{i}>: i<\kappa\right\rangle
$$

where $\zeta_{i}<\mu_{i}$ and each $B_{i, \zeta}$ is a non-empty subset of $f(i)+1$

(ii) $\left|\mathscr{P}^{*}\right|<\lambda^{*}$

(iii) if $\left\langle B_{i, \zeta}: i<\kappa, \zeta<\zeta_{i}\right\rangle \in \mathscr{P}^{*}, g \in \prod_{i<\kappa}(f(i)+1), A \subseteq \kappa$ and for $i \in A$ we have $\xi_{i}<\zeta_{i}$ satisfying $g(i) \in B_{i, \xi_{i}}$ then there are $E,\left\langle A_{j}: j<\kappa\right\rangle$ and for $j<\kappa$ sequences $\bar{B}^{j}=\left\langle B_{i, \zeta}^{j}: i<\kappa, \zeta<\zeta_{i}^{j}\right\rangle$ and $\left\langle\xi_{i}^{j}: i \in A_{j}\right\rangle$ such that:

(a) $E \subseteq \kappa$ and $\kappa \backslash E \in J$;

(b) $A \cap E=\left\{i<\kappa: i \in \bigcup_{j<i} A_{j}\right\}$;

(c) $\bar{B}^{j} \in \mathscr{P}^{*}$ for $j<\kappa$;

(d) for $j<\kappa$ and $i \in A_{j}$ we have: $\xi_{i}^{j}<\zeta_{i}^{j}$ and $B_{i,\left(\xi_{i}^{j}\right)}^{j} \subseteq B_{i, \xi_{i}}$ and

$$
\left[\left|B_{i,\left(\xi_{i}^{j}\right)}^{i}\right| \geq \mu_{i} \Rightarrow\left|B_{i,\left(\xi_{i}^{j}\right)}^{j}\right|<\left|B_{i,\left(\xi_{i}^{j}\right)}\right|\right]
$$

(e) for $i \in A_{j}$ we have $g(i) \in B_{i,\left(\xi_{i}^{j}\right)}^{j}$ 
Proof of the Inequality from the Fact. Let us define a family $\mathscr{P}^{\prime}$ :

$$
\mathscr{P}^{\prime}=\left\{\left\langle\cup\left\{B_{i, \zeta}: \zeta<\zeta_{i} \text { and }\left|B_{i, \zeta}\right|<\mu_{i}\right\}: i<\kappa\right\rangle:\left\langle B_{i, \zeta}: i<\kappa, \zeta<\zeta_{i}\right\rangle \in \mathscr{P}^{*}\right\} .
$$

Now each member of $\mathscr{P}^{\prime}$ has the right form as each $\mu_{i}$ is regular and $\left\langle B_{i, \zeta}: i<\right.$ $\left.\kappa, \zeta<\zeta_{i}\right\rangle \in \mathscr{P}^{*}$ implies $\zeta_{i}<\mu_{i}$. Also the cardinality of $\mathscr{P}^{\prime}$ is $<\lambda^{*}$ (by (ii) of 1.3).

Let $g \leq f$ and it is enough to find $\left\langle B_{i}^{\epsilon}: i<\kappa\right\rangle \in \mathscr{P}^{\prime}$ for $\epsilon<\kappa$ such that $\left\{i: g(i) \notin \bigcup_{\epsilon<i} B_{i}^{\varepsilon}\right\} \in J$.

We choose by induction on $n$, for every $\eta \in{ }^{n} \kappa$ the following: $\bar{B}^{\eta}=\left\langle B_{i, \zeta}^{\eta}: i<\right.$ $\left.\kappa, \zeta<\zeta_{i}^{\eta}\right\rangle \in \mathscr{P}^{*},\left\langle\xi_{i}^{\eta}: i \in A_{\eta}\right\rangle$ with $\xi_{i}^{\eta}<\zeta_{i}^{\eta}$ and $A_{\eta} \subseteq \kappa$ such that:

( $\alpha) \bar{B}^{<>}=\langle<f(i)+1>: i<\kappa\rangle, \zeta_{i}^{<>}=1, \xi_{i}^{<>}=0, A_{<>}=\kappa$,

$(\beta)\left[i \in A_{\eta^{\wedge}<j>} \Rightarrow g(i) \in B_{i,\left(\xi_{i}^{\eta^{\wedge}<j>}\right)}^{\eta^{\wedge}<j>} \subseteq B_{i, \xi_{i}^{\eta}}^{\eta}\right]$,

$(\gamma)\left[i \in A_{\eta^{\wedge}<j>} \&\left|B_{i, \xi_{i}^{\eta}}^{\eta}\right| \geq \mu_{i} \Rightarrow\left|B_{i,\left(\xi_{i}^{\eta^{\wedge}<j>}\right)}^{\eta^{\wedge}<j>}\right|<\left|B_{i, \xi_{i}^{\eta}}^{\eta}\right|\right]$ and

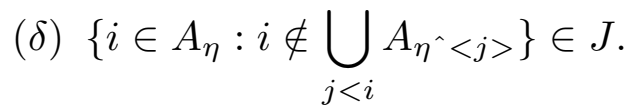

The induction step is by (iii) of the fact; in the end let for $\eta \in{ }^{\omega>} \kappa$ and $i<\kappa$ : $B_{\eta, i}^{*}=: \cup\left\{B_{i, \zeta}^{\eta}: \zeta<\zeta_{i}^{\eta}\right.$ and $\left.\left|B_{i, \zeta}^{\eta}\right|<\mu_{i}\right\}$.

Clearly for each $\eta \in{ }^{\omega>} \kappa$ we have $\left\langle B_{\eta, i}^{*}: i<\kappa\right\rangle \in \mathscr{P}^{\prime}$; let us enumerate $\omega_{>} \kappa$ as $\left\{\rho_{\epsilon}: \epsilon<\kappa\right\}$ such that $\left[\rho \triangleleft \rho_{\epsilon} \Rightarrow \rho \in\left\{\rho_{\zeta}: \zeta<\epsilon\right\}\right]$, and let us define $\bar{B}^{\epsilon}=\left\langle B_{\rho_{\epsilon}, i}^{*}: i<\kappa\right\rangle \in \mathscr{P}^{\prime}$, hence by Definition 1.1 it is enough to show that $E=:\left\{i<\kappa: g(i) \notin \bigcup_{\epsilon<i} B_{\rho_{\epsilon}, i}^{*}\right\}$ belongs to $J$. We know that for every $\eta \in{ }^{\omega>} \kappa$ the set $X_{\eta}=:\left\{i \in A_{\eta}: i \notin \bigcup_{j<i} A_{\eta^{\wedge}<j>}\right\}$ belongs to $J$. Also the sets

$$
\begin{gathered}
Y=:\left\{\delta<\kappa: \delta \text { limit and } \neg(\forall \epsilon)\left[\epsilon<\delta \Rightarrow \rho_{\epsilon} \in{ }^{\omega>} \delta\right]\right\} \\
X=:\left\{i<\kappa: \text { if } i \notin Y \text { then for some } \eta \in^{\omega>} i \text { we have } i \in X_{\eta}\right\}
\end{gathered}
$$

belong to $J$. It suffices to show

(*) for every $\delta \in \kappa \backslash X$, for some $\eta \in{ }^{\omega>} \delta$ we have $\delta \in B_{\eta, i}^{*}$.

Why $(*)$ holds? Choose by induction on $n<\omega, \rho_{n} \in{ }^{n} \delta$ such that: $\delta \in A_{\rho_{n}}$. For $n=0$ remember $A_{<>}=\kappa$. For $n+1$, as $\delta \in A_{\rho_{n}}$ and $\delta \notin X$ clearly $\delta \notin X_{\rho_{n}}$ so necessarily $\delta \in \bigcup_{j<i} A_{\rho_{n^{\wedge}<j>}}$.

Now $\left\langle\left|B_{\delta, \xi_{\delta}^{\rho_{n}}}^{\rho_{n}}\right|: n<\omega\right\rangle$ is non-increasing hence (by $(\beta)$ above) is eventually constant hence (by $(\gamma)$ above) for some $n,\left|B_{\delta, \xi_{\delta}^{\rho_{n}}}^{\rho_{n}}\right|<\mu_{\delta}$, hence $g(\delta) \in B_{\rho_{n}, \delta}^{*}$. So $(*)$ holds and we have finished proving that $\mathscr{P}^{\prime}$ exemplify the inequality from the fact.

Proof of the Fact 1.1A. It suffices to prove that for any $\bar{B}^{*}=\left\langle B_{i, \zeta}^{*}: i<\kappa, \zeta<\zeta_{i}\right\rangle$ 
for the given $\bar{B}^{*}$ and any $g,\left\langle\xi_{i}: i<\kappa\right\rangle$ as there. Let $Y_{0}^{i}=\left\{\zeta<\zeta_{i}:\left|B_{i, \zeta}^{*}\right|<\right.$ $\left.\mu_{i}\right\}, Y_{1}^{i}=\left\{\zeta<\zeta_{i}:\left|B_{\zeta, i}^{*}\right| \geq \mu_{i}>\operatorname{cf}\left|B_{\zeta, i}^{*}\right|\right\}$ and $Y_{2}^{i}=\left\{\zeta<\zeta_{i}: \operatorname{cf}\left|B_{i, \zeta}^{*}\right| \geq \mu_{i}\right\}$ (for each $\left.i<\kappa, \zeta<\zeta_{i}\right)$.

Clearly $\left\langle Y_{0}^{i}, Y_{1}^{i}, Y_{2}^{i}\right\rangle$ is a partition of $\left\{\zeta: \zeta<\zeta_{i}\right\}$, now for $\zeta \in Y_{1}^{i} \cup Y_{2}^{i}$, let $\mu_{\zeta}^{i}=: \quad \mathrm{cf}\left|B_{\zeta, i}^{*}\right|$, and $\left\langle B_{i, \zeta, \epsilon}: \epsilon<\mu_{\zeta}^{i}\right\rangle$ be an increasing continuous sequence of subsets of $B_{i, \zeta}^{*}$ of cardinality $<\left|B_{\zeta, i}^{*}\right|$ and $\bigcup_{\epsilon<\mu_{\zeta}^{i}} B_{i, \zeta, \epsilon}=B_{i, \zeta}^{*}$.

Now let $\mathfrak{a}=:\left\{\mu_{\zeta}^{i}: i<\kappa, \zeta \in Y_{2}^{i}\right\}$, so $\mathfrak{a}$ is a set of regular cardinals.

Case 1: Assume assumption $(\alpha)$ of 1.2 ;

so $\mathfrak{a}$ is a set of $\left\langle\mu+\kappa^{+}=\mu\right.$ regular cardinals, each $\geq \mu$, so the pcf analysis of [Sh $371, \S 2]$ apply. Let us get $\left\langle\left\langle f_{\alpha}^{\theta}: \alpha<\theta\right\rangle: \theta \in \operatorname{pcf}(\mathfrak{a})\right\rangle$.

Now for each $\theta \in \operatorname{pcf}(\mathfrak{a}) \cup\{1\}$ which is $<\lambda^{*}$ and $\alpha<\theta$, we choose $\bar{B}^{\theta, \alpha}=$ $\left\langle B_{i, \zeta}^{\theta, \alpha}: i<\kappa, \zeta<\zeta_{i}^{\theta, \alpha}\right\rangle$ such that:

$$
\begin{aligned}
\left\{B_{i, \zeta}^{\theta, \alpha}: \zeta<\zeta_{i}^{\theta, \alpha}\right\}= & \left\{B_{i, \zeta}^{*}: \zeta \in Y_{0}^{i}\right\} \cup\left\{B_{i, \zeta, \epsilon}^{*}: \epsilon<\mu_{\zeta}^{i}, \zeta \in Y_{1}^{i}\right\} \\
& \cup\left\{B_{i, \zeta, f_{\alpha}^{\theta}\left(\mu_{\zeta}^{i}\right)}: \zeta \in Y_{2}^{i} \text { and } \theta \in \operatorname{pcf}(\mathfrak{a})(\text { i.e. } \theta \neq 1)\right\}
\end{aligned}
$$

Let $\mathscr{P}_{\bar{B}^{*}}=:\left\{\bar{B}^{\theta, \alpha}: \theta \in \lambda^{*} \cap \operatorname{pcf}(\mathfrak{a})\right.$ and $\alpha<\theta$ or $\left.\theta=1, \alpha=0\right\}$. Now it is as required, in particular $\left|\mathscr{P}_{\bar{B}^{*}}\right|<\lambda^{*}$ because $\lambda^{*}>\sup \left(\lambda^{*} \cap \operatorname{pcf}(\mathfrak{a})\right.$ holds as $\lambda^{*}$ is a successor cardinal.

Case 2: Assume assumption. Here we partition $\mathfrak{a}$ to $\kappa$ sets diagonally; i.e. without loss of generality each $\zeta_{i}$ is a cardinal hence by clause $(\beta)$ we have $\zeta_{i} \leq \bigcup_{j<i} \mu_{j+1}+\mu_{0}$. So for every limit $i<\kappa$ we have $\zeta_{i} \leq \bigcup_{j<i} \mu_{j}$. Remember $\kappa<\bigcup_{i<\kappa} \mu_{i}$ and even $\kappa<\mu_{0}$ and let for $j<\kappa$ :

$\mathfrak{a}_{j}=:\left\{\mu_{\zeta}^{i}: i \in(j, \kappa)\right.$ and $\zeta \in Y_{2}^{i}$ and $\left.\zeta<\mu_{j}\right\}$. So $\left|\mathfrak{a}_{j}\right| \leq \kappa+\mu_{j}<\operatorname{Min}\left(\mathfrak{a}_{j}\right)$ because and relevant $\mu_{\zeta}^{i}$ is $\geq \mu_{i}>\mu_{j} \geq \kappa$ and if $\left\langle\xi_{i}: i<\kappa\right\rangle \in \prod_{i<\kappa}\left(1+\zeta_{i}\right)$ then we can define $h: \kappa \rightarrow \kappa, h(i)<1+i$ such that: $\left[i<\kappa \& i\right.$ limit $\left.\Rightarrow \mu_{\xi_{i}}^{i} \in \mathfrak{a}_{h(i)}\right] . \quad \square_{1.2}$

The following lemma generalizes [Sh 371, 1.5].

1.5 Lemma. Suppose $\sigma_{1} \leq \sigma_{2} \leq \kappa<\theta<\lambda$ are cardinals, $\sigma_{1}, \sigma_{2}, \kappa$ are regular, $\lambda>c f(\lambda)=\kappa>\aleph_{0}, \lambda<\mu=c f(\mu)<p p_{\Gamma\left(\theta, \sigma_{2}\right)}^{+}(\lambda)$, and for every large enough $\lambda^{\prime}<\lambda,\left[\sigma_{1} \leq c f\left(\lambda^{\prime}\right)<\theta \Rightarrow p p_{\Gamma\left(\theta, \sigma_{1}\right)}\left(\lambda^{\prime}\right)<\lambda\right]$.

Then there is an increasing sequence $\left\langle\mu_{i}: i<\kappa\right\rangle$ of regular cardinals $<\lambda, \lambda=$ $\sup _{i<\kappa} \mu_{i}$ and an ideal $J$ on $\kappa$ satisfying $\lambda=\operatorname{tlim}_{J} \mu_{i}$ and $\mu=t c f\left(\prod_{i<\kappa} \mu_{i} / J\right)$ such that:

(a) $J$ is $\sigma_{2}$-complete and extend $J_{\kappa}^{\mathrm{bd}}$

(b) if $\kappa \geq \sigma_{1}^{+}$and $(\forall \alpha<\kappa)\left[\operatorname{cov}\left(|\alpha|, \sigma_{2}, \sigma_{1}, 2\right)<\kappa\right]$ then $J$ is normal; 
Proof. For $(c)$ see $[$ Sh $371,1.6]$, so we can assume $\sigma_{1}>\aleph_{0}$ as otherwise we have there gotten a conclusion stronger than $(\mathrm{a})+(\mathrm{b})+(\mathrm{c})$.

Let $\mathfrak{a} \subseteq \operatorname{Reg} \cap \lambda$ have cardinality $<\theta$, be unbounded in $\lambda, I$ a $\sigma_{2}$-complete ideal on $a$, and $\left(\forall \lambda^{\prime}<\lambda\right)\left[\mathfrak{a} \cap \lambda^{\prime} \in I\right]$ and $\mu=\operatorname{tcf}(\Pi \mathfrak{a} / I)$. As $\operatorname{cf}(\lambda)=\kappa<\theta<\lambda$ without loss of generality $\theta<\operatorname{Min}(\mathfrak{a})$, and let $\left\langle\lambda_{i}: i<\kappa\right\rangle$ be increasing continuous with limit $\lambda$, $\aleph_{0} \leq \operatorname{cf}\left(\lambda_{i}\right)<\kappa$ (remember $\kappa>\aleph_{0}$ ); without loss of generality $\left.\left.\theta<\lambda_{0}<\operatorname{Min}\right) \mathfrak{a}\right)$ and $\operatorname{pp}_{\Gamma\left(\theta, \sigma_{1}\right)}\left(\lambda_{i}\right)<\lambda_{i+1}$ and, if $i>0$ is a limit ordinal then $\mathfrak{a} \cap \lambda_{i}$ is unbounded in $\lambda_{i}$. Also without loss of generality for every $i<\kappa$ :

$$
(*)_{1} \quad \lambda_{0} \leq \lambda^{\prime}<\lambda_{i} \& \sigma_{1} \leq \operatorname{cf}\left(\lambda^{\prime}\right)<\theta \Rightarrow \operatorname{pp}_{\Gamma\left(\theta, \sigma_{1}\right)}\left(\lambda^{\prime}\right)<\lambda_{i+1}
$$

hence

$$
(*)_{2} \mathfrak{b} \subseteq\left(\lambda_{0}, \lambda_{i}\right) \&|\mathfrak{b}|<\theta \Rightarrow \operatorname{sup~pcf}_{\sigma_{1} \text {-complete }}(\mathfrak{b})<\lambda_{i+1} .
$$

Let $\left\langle\mathfrak{b}_{\sigma}[\mathfrak{a}]: \sigma \in \operatorname{pcf}(\mathfrak{a})\right\rangle$ be a generating sequence (exists by [Sh 371, 2.6]) and without loss of generality $\mu=\max \operatorname{pcf}(\mathfrak{a})$. By [Sh 345a, 3.6] (and 3.1(7)) without loss of generality $\sigma \in \mathfrak{b}_{\chi}[\mathfrak{a}] \Rightarrow \mathfrak{b}_{\sigma}[\mathfrak{a}] \subseteq \mathfrak{b}_{\chi}[\mathfrak{a}]$. Let $i<\kappa$ satisfy $\operatorname{cf}(i) \geq \sigma_{1}$, as $|\mathfrak{a}|<\theta$ and $(*)_{1}$ we have $\sup \operatorname{pcf}_{\sigma_{1} \text {-complete }}\left(\lambda_{i} \cap \mathfrak{a}\right)<\lambda_{i+1}$ hence for some $\mathfrak{c}_{i}$ we have: $\mathfrak{c}_{i} \subseteq \lambda_{i+1} \cap \operatorname{pcf}\left(\mathfrak{a} \cap \lambda_{i}\right),\left|\mathfrak{c}_{i}\right|<\sigma_{1}$ and $\mathfrak{a} \cap \lambda_{i} \subseteq \bigcup_{\theta \in \mathfrak{c}_{i}} \mathfrak{b}_{\theta}[\mathfrak{a}]$ (otherwise we can find a $\sigma_{1}$-complete proper ideal $J$ on $\lambda_{i} \cap \mathfrak{a}$ such that

$$
\left[\sigma<\operatorname{pp}_{\Gamma\left(\theta, \sigma_{1}\right)}^{+}\left(\lambda_{i}\right) \& \sigma \in \operatorname{pcf}\left(\lambda_{i} \cap \mathfrak{a}\right) \Rightarrow \mathfrak{b}_{\sigma}[\mathfrak{a}] \cap\left(\lambda_{i} \cap \mathfrak{a} \backslash \mu_{i}\right) \in J\right]
$$

a contradiction to $(*)_{1}$. Note that $\mathfrak{c}_{i} \subseteq \lambda_{i+1}$.

Let $S_{0}=\left\{\delta<\kappa: \operatorname{cf}(\delta) \geqq \sigma_{1}\right\}$, so for some $i(*)<\kappa$ we have:

$$
S_{1}=\left\{\delta \in S_{0}: \mathfrak{c}_{\delta} \cap \lambda_{\delta} \subseteq \lambda_{i(*)}\right\}
$$

is a stationary subset of $\kappa$.

By renaming, without loss of generality $i(*)=0$ and so $\mathfrak{c}_{i} \subseteq\left(\lambda_{i}, \lambda_{i+1}\right)$, and for $i \in S_{1}$ let $\left\langle\left(\theta_{i, \zeta}, \mathfrak{e}_{i, \zeta}\right): \zeta<\zeta_{i}\right\rangle$ list $\left\{\left(\theta, \mathfrak{b}_{\theta}[\mathfrak{a}]\right): \theta \in \mathfrak{c}_{i}\right\}$; so:

$$
\begin{aligned}
(*)_{1} \mathfrak{a} \cap \lambda_{i} \subseteq \bigcup_{\zeta<\zeta_{i}} \mathfrak{e}_{i, \zeta}, \max \operatorname{pcf}\left(\mathfrak{e}_{i, \zeta}\right)=\theta_{i, \zeta}, \lambda_{i}<\theta_{i, \zeta}<\lambda_{i+1} \\
\quad \text { and } \theta_{i, \zeta} \in \operatorname{pcf}_{\sigma \text {-complete }}\left(\mathfrak{a} \cap \lambda_{i}\right) .
\end{aligned}
$$

1.6 Fact. There are finite $\mathfrak{d}_{i, \zeta} \subseteq \operatorname{pcf}\left(\mathfrak{e}_{i, \zeta}\right) \backslash \lambda_{i}$ for $i \in S_{1}, \zeta<\zeta_{i}$ and stationary $S_{2} \subseteq S_{1}$ such that letting $\mathfrak{d}_{i}=\bigcup_{\zeta<\zeta_{i}} d_{i, \zeta}$ we have: if $S \subseteq S_{2}, \kappa=\sup (S)$ then $\mu \in \operatorname{pcf}_{\sigma_{2} \text {-complete }}\left(\bigcup_{i \in S} \mathfrak{d}_{i}\right)$

Proof of 1.2 from the Fact. Now the preliminary part of 1.5 is easy; as $\mathfrak{d}_{i} \subseteq$ $\left(\lambda_{i}, \lambda_{i+1}\right)$, and $\left|\mathfrak{d}_{i}\right|<\sigma_{1}<\kappa=\operatorname{cf}(\kappa)$, clearly $\mathfrak{d}=: \bigcup \mathfrak{d}_{i}$ has order type $\kappa$ 
and by $1.6 \mu \in \operatorname{pcf}_{\sigma_{2} \text {-complete }}\left(\bigcup_{i \in S_{1}} \mathfrak{d}_{i}\right)$ and by $(*)_{2}$ above, for $j<\kappa$ implies $\mu \notin \operatorname{pcf}_{\sigma_{1} \text {-complete }}\left(\bigcup_{i \in S_{1}} \mathfrak{d}_{i} \cap \lambda_{j}\right)$; also $\mu=\max \operatorname{pcf}(\mathfrak{d})$. So we are left with clauses $(a)+(b)$. Let $\mathfrak{d}_{\delta}=\left\{\lambda_{\delta, \zeta}: \zeta<\zeta_{\delta}<\sigma_{1}\right\}$, as $\sigma_{1}<\kappa$, clearly without loss of generality for some $\zeta(*)$

$$
S_{3}=\left\{\delta \in S_{2}: \zeta_{\delta}=\zeta(*)\right\} \text { is stationary. }
$$

For each $\zeta<\zeta(*)$ let

$$
\mathscr{P}_{\zeta}=:\left\{S \subseteq S_{2}: \max \operatorname{pcf}\left\{\lambda_{\delta, \zeta}: \delta \in S\right\}<\mu\right\}
$$

If for some $\zeta<\zeta(*)$, the normal ideal on $\kappa$ which $\mathscr{P}_{\zeta}$ generates is proper, we have finished. If not, for each $\zeta<\zeta(*)$ there are members $S_{\zeta, i}(i<\kappa)$ of $\mathscr{P}_{\zeta}$ and club $C_{\zeta}$ of $\kappa$ such that:

$$
\delta \in S_{3} \cap C_{\zeta} \Rightarrow \bigvee_{i<\delta} \delta \in S_{\zeta, i}
$$

Clearly $C=\bigcap_{\zeta<\zeta(*)} C_{\zeta}$ is a club of $\kappa$, now remembering $S_{3} \subseteq S_{0}$ we know that on $S_{3} \cap C$ the function $\delta \mapsto \sup \left\{\operatorname{Min}\left\{i: \delta \in S_{\zeta, i}\right\}: \zeta<\zeta(*)\right\}$ is a pressing down function, so for some stationary $S_{4} \subseteq S_{3}$ and ordinal $j(*)<\operatorname{Min}\left(S_{4}\right)<\kappa$ we have:

$$
\delta \in S_{4} \Leftrightarrow \bigwedge_{\zeta<\zeta(*)} \bigvee_{j<j(*)} \delta \in C \cap S_{\zeta, j}
$$

But as $\operatorname{cov}\left(|j(*)|, \sigma_{2}, \sigma_{1}, 2\right)<\kappa$, there is $w \subseteq j(*)$ such that $|w|<\sigma_{2}$ and $S_{5}$ is a stationary subset of $\kappa$ where

$$
S_{5}=\left\{\delta \in S_{3}: \bigwedge_{\zeta<\zeta(*)} \bigvee_{i \in w} \delta \in S_{\zeta, i}\right\}
$$

Let $\mathfrak{b}=\left\{\lambda_{i, \zeta}\right.$ : for some $j$ and $\zeta$ we have $i \in S_{\zeta, j}, j \in w$ and $\left.\zeta<\zeta(*)\right\}$, it is the union of $<\sigma_{2}$ sets $\left(\mathfrak{b}_{j, \zeta}=\left\{\lambda_{i, \zeta}: i \in S_{\zeta, j}\right\}\right.$ for $\left.j \in w, \zeta<\zeta(*)\right)$, each with max pcf $<\mu$.

This contradicts the fact (1.6).

Proof of Fact 1.2A. Similar to the proof of [Sh 371, 1.5]. 
SAHARON: on 2.1 see 430 ?

Improving a little [Sh 400, 5.9].

2.1 Claim. Assume $\lambda>\operatorname{cf}(\lambda)=\aleph_{0}, \lambda>2^{\theta}$ and $\left[\lambda^{\prime}<\lambda \quad \& \quad \operatorname{cf}\left(\lambda^{\prime}\right) \leqq \theta \Rightarrow\right.$ $\left.\operatorname{pp}_{\theta}\left(\lambda^{\prime}\right)<\lambda\right]$ and $\operatorname{pp}(\lambda)<\operatorname{cov}\left(\lambda, \lambda, \aleph_{1}, 2\right)$. Then $\left\{\mu: \lambda<\mu=\aleph_{\mu} \leq \operatorname{pp}_{\theta}(\lambda)\right\}$ has order type $\geq \theta$.

We shall return to this in [Sh 430, §1] so we do not elaborate.

2.2 Claim. Suppose $\theta, \kappa$ are regular, $\aleph_{0}<\theta \leq \kappa<\lambda \leq \lambda_{1} \leq \lambda^{*}$, and $(\forall \mu)[\lambda \leq$ $\left.\mu \leq \lambda_{1} \& \operatorname{cf}(\mu)<\theta \Rightarrow \operatorname{pp}_{<\theta}(\mu) \leqq \lambda^{*}\right]$ and $\operatorname{cov}(\kappa, \theta, \theta, 2)=\kappa$. Then there is a family $\mathscr{P}$ of $\leq \lambda^{*}$ subsets of $\lambda_{1}$, each of cardinality $<\lambda$ such that:

$(*)_{1}$ for every $Y \subseteq \lambda_{1},|Y| \leq \kappa$ there are $Z_{n}(n<\omega)$ such that:

$$
\begin{aligned}
& Y \subseteq \bigcup_{n<\omega} Z_{n},\left|Z_{n}\right|=\kappa \text { and for each } n \\
& (\forall Z)\left[Z \subseteq Z_{n} \quad \&|Z|<\theta \Rightarrow(\exists X \in \mathscr{P})[Z \subseteq X]\right],
\end{aligned}
$$

$(*)_{2}$ for every $Y \subseteq \lambda_{1},|Y| \geq \theta_{1}$ and $\aleph_{1}<\operatorname{cf}\left(\theta_{1}\right)$ \& $\theta_{1} \leq \theta$ there is $X \in \mathscr{P}$ such that: $X \cap Y$ has cardinality $\geq \theta_{1}$.

Remark. 1) Here and later we can replace $\leq \lambda^{*}$ by $<\lambda^{*}=\operatorname{cf}\left(\lambda^{*}\right)$. [Saharon: check.] 2) See [Sh 430, x.x.].

Proof. It suffices to prove $(*)_{1}$ as $(*)_{2}$ follows. Let $\Theta=\left\{\mu: \lambda \leq \mu \leq \lambda_{1}\right.$ and $\operatorname{cf}(\mu)<\theta\}$. Clearly if $\Theta=\emptyset$, the conclusion is straightforward (by induction on $\left.\lambda_{1}\right)$.

Without loss of generality $\lambda^{*}=\sup \left\{\operatorname{pp}_{<\theta}(\mu): \mu \in \Theta\right\}$. Now each $\operatorname{pp}_{<\theta}(\mu)$ (for $\mu \in \Theta)$ has cofinality $\geq \theta$, and if $\delta<\theta,\left\langle\mu_{i}: i<\delta\right\rangle$ increasing, $\left\langle\operatorname{pp}_{<\theta}\left(\mu_{i}\right): i<\delta\right\rangle$ strictly increasing then $\operatorname{pp}_{<\theta}\left(\bigcup_{i<\delta} \mu_{i}\right)>\sum_{i<\delta} \operatorname{pp}_{<\theta}\left(\mu_{i}\right)$, hence $\operatorname{cf}\left(\lambda^{*}\right) \geq \theta$ and, by [Sh 355, 2.3], without loss of generality $\lambda^{*}=\lambda_{1}$. Let $\chi$ be regular large enough and $B$ be an elementary submodel of $\left(\mathscr{H}(\chi), \in,<_{\chi}^{*}\right)$ of cardinality $\lambda^{*}$ such that $\lambda^{*}+1 \subseteq B$. Let

$$
\mathscr{P}=\left\{X \in B: X \subseteq \lambda^{*} \text { and }|X|<\lambda\right\} .
$$

Now repeat the proof of [Sh 400, 3.5], noting

2.3 Observation. Suppose: 
(ii) the function $\mathfrak{b} \mapsto\left\langle f_{\alpha}^{\mathfrak{b}}: \alpha<\max \operatorname{pcf}(\mathfrak{b})\right\rangle($ for $\mathfrak{b} \subseteq \mathfrak{a})$ is as in [Sh 371, §1]; i.e. satisfies:

$(*)_{1} \quad f_{\alpha}^{\mathfrak{b}} \in \Pi \mathfrak{b}$, we stipulate $f_{\alpha}^{b} \uparrow(\mathfrak{a} \backslash \mathfrak{b})=0_{\mathfrak{a} \backslash \mathfrak{b}}$ and $\left\langle f_{\alpha}^{\mathfrak{b}}: \alpha<\theta\right\rangle$ is

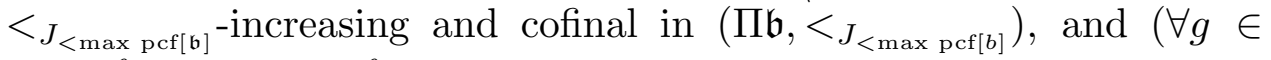
$\Pi \mathfrak{b})\left(\exists^{\theta} \alpha<\theta\right)\left[g<f_{\alpha}^{\theta}\right]$

$(*)_{2} \quad$ if $\delta<\theta \in \operatorname{pcf}(\mathfrak{a}), \operatorname{cf}(\delta) \in(|\mathfrak{b}|, \operatorname{Min} \mathfrak{b}]$ and $\sigma \in \mathfrak{a} \backslash\{\operatorname{cf}(\delta)\}$ then $f_{\delta}^{\mathfrak{b}}(\sigma)=$ $\operatorname{Min}\left\{\bigcup_{\alpha \in C} f_{\alpha}^{\mathfrak{b}}(\sigma): C\right.$ a club of $\left.\delta\right\}$

(iii) $\mathfrak{a} \subseteq \lambda^{*}, \lambda \leq \lambda^{*}$, and

$(\forall \mathfrak{b})\left[\mathfrak{b} \subseteq \mathfrak{a} \quad \&|\mathfrak{b}|<\theta \quad \& \sup (\mathfrak{b}) \geq \lambda \quad \& \quad \sup (\mathfrak{b}) \notin \mathfrak{b} \Rightarrow \max \operatorname{pcf}_{J_{\mathfrak{b}}^{b d}} \mathfrak{b} \leq \lambda^{*}\right]$

(iv) We let for $\theta \in \operatorname{pcf}(\mathfrak{a})$, and $\alpha<\theta: f_{\alpha}^{\theta}=f_{\alpha}^{\mathfrak{b}_{\theta}[\mathfrak{a}]}$.

Then for every $\left\{\mathfrak{a}_{i}: i<\kappa\right\} \subseteq\{\mathfrak{b}: \mathfrak{b} \subseteq \mathfrak{a},|\mathfrak{b}|<\theta\}, g_{i} \in \Pi_{i}(i<\kappa)$ we can find $g$ and $\lambda_{i}^{\prime}<\lambda$ such that: $g \in \Pi \mathfrak{a}$, for each $i<\kappa, g_{i} \leq g$ and for every $i<\kappa$ there are $\lambda_{i}^{\prime}<\lambda$ such that letting $\mathfrak{c}^{i}=\mathfrak{a}^{i} \backslash \lambda_{i}^{\prime}$ we have $g\left\lceil\mathfrak{c}^{i}\right.$ is Max of finitely many functions from $\left\{f_{\alpha}^{\mu}\left\lceil c^{i}: i<\kappa, \alpha<\mu, \mu \in \operatorname{pcf}(\mathfrak{a})\right.\right.$ and $\left.\mu \leq \lambda^{*}\right\}$. Moreover for some $\mathfrak{d} \subseteq\left[\lambda, \lambda^{*}\right] \cap \operatorname{pcf}(\mathfrak{a})$, for every $\theta \in \operatorname{Reg} \cap\left[\lambda, \lambda^{*}\right] \cap \mathfrak{d}$, for some $\lambda_{\theta}<\lambda, g\left\lceil\left(\mathfrak{b}_{\theta}[\mathfrak{a}] \backslash \lambda_{\theta}\right)\right.$ is (the suitable restriction of $a$ ) Max of finitely many functions from $\left\{f_{g(\sigma)}^{\sigma}: \sigma \in\left(\lambda, \lambda^{*}\right] \cap \operatorname{pcf}\left(\mathfrak{a} \backslash \lambda^{\prime}\right)\right.$ for every $\left.\lambda^{\prime}<\lambda\right\}$.

Proof. Without loss of generality $\kappa^{+}<\operatorname{Min}(\mathfrak{a})$. Use [Sh 371, 1.4] with $\kappa^{+}, \mathfrak{a},\left\langle\mathfrak{a}_{i}\right.$ : $i<\kappa\rangle$, here standing for $\delta, \mathfrak{a},\left\langle\mathfrak{b}_{i}: i\left\langle\zeta^{*}\right\rangle\right.$ there and we get $\left\langle\left\langle\lambda_{i, \ell}, \mathfrak{c}_{i, \ell}: \ell \leq n(i)\right\rangle\right.$ : $i<\kappa\rangle$. Let $\lambda_{i}^{\prime}=\sup \left[\cup\left\{\mathfrak{c}_{i, \ell}: \mathfrak{c}_{i, \ell}\right.\right.$ a bounded subset of $\left.\left.\lambda\right\}\right]$.

Similarly

2.4 Claim. 1) In 2.2, if $\theta=\kappa$ and $(\forall \mu)\left[\kappa \leq \mu \leq \lambda_{1} \quad \& \quad \operatorname{cf}(\mu)<\theta \Rightarrow \operatorname{pp}_{<\theta}(\mu) \leq\right.$ $\lambda^{*}$ ] (i.e. $\kappa=\lambda$ ) then we can add

$(*)_{3}$ for every $Y \subseteq \lambda,|Y| \leq \kappa$ there are $Z_{n},\left\langle Z_{n, i}: i<\theta\right\rangle$ for $n<\omega$ such that $Y \subseteq \bigcup_{n<\omega} Z_{n}, Z_{n}=\bigcup_{i<\theta} Z_{n, i},\left|Z_{n, i}\right|<\theta,\left\langle Z_{n, i}: i<\theta\right\rangle$ increasing continuous and each $Z_{n, i}$ belongs to $\mathscr{P}$.

Hence

2.5 Conclusion. If $\lambda>\operatorname{cf}(\lambda)=\aleph_{0} \underline{\text { then }}$ there is a family $\mathscr{P}$ of cardinality $\leq$ $\sup \left\{p p(\mu): \mu \leq \lambda, \operatorname{cf}(\mu)=\lambda_{0}\right\}$ consisting of countable subsets of $\lambda$ such that:

(*) if $Y \subseteq \lambda,|Y|=\aleph_{1}$ then for some $Z \in \mathscr{P}, Y \cap Z$ is infinite. Moreover, we can find $\alpha_{i}^{n}\left(n<\omega, i<\omega_{1}\right)$ such that $Y \subseteq\left\{\alpha_{i}^{n}: n<\omega, i<\omega_{1}\right\}$ and for each $n$ for arbitrarily large $i<\omega_{1},\left\{\alpha_{j}^{n}: j<i\right\} \in \mathscr{P}$.

2.6 Conclusion. If $\lambda>\kappa \geq \operatorname{cf}(\lambda)$, and $\operatorname{pp}_{\kappa}(\lambda)<\operatorname{cov}\left(\lambda, \lambda, \kappa^{+}, 2\right)$ then $\operatorname{pp}_{\kappa}(\lambda)<$ 
Proof. Let $\chi=\beth_{3}(\lambda)^{+}$, and for $\zeta \leq \kappa^{+}$let $\mathfrak{B}_{\zeta}$ be an elementary submodel of $\left(\mathscr{H}(\chi), \in,<_{\chi}^{*}\right)$ of cardinality $\operatorname{pp}_{\kappa}(\lambda)$ such that $\operatorname{pp}_{\kappa}(\lambda) \subseteq \mathfrak{B}_{\zeta}, \mathfrak{B}_{\zeta}$ increasing continuous, and $\left\langle\mathfrak{B}_{\epsilon}: \epsilon \leqq \zeta\right\rangle \in \mathfrak{B}_{\zeta+1}$. Let $\mathfrak{B}=\mathfrak{B}_{\kappa^{+}}$. Assume that the conclusion fail and we shall prove that $\operatorname{cov}\left(\lambda, \lambda, \kappa^{+}, 2\right) \leqq \operatorname{pp}_{\kappa}(\lambda)$, in fact that $\mathscr{P}=: \mathfrak{B}_{\kappa^{+}} \cap \mathscr{S}_{<\lambda}(\lambda)$ exemplify it. Let $a \subseteq \lambda,|a| \leq \kappa$ and we shall find $A \in \mathscr{P}$ such that $a \subseteq A$; this suffice. Choose by induction on $\zeta<\omega_{1}, f_{\zeta} \in \mathfrak{B} \cap \Pi(\operatorname{Reg} \cap \lambda)$ such that letting $N_{\zeta}$ be the Skolem Hull of $a \cup\left\{f_{\xi}: \xi<\zeta\right\} \cup\{i: i \leq \kappa\}$, we have: for every large enough $\sigma \in \operatorname{Reg} \cap \lambda,\left[\sigma \in N_{\zeta} \Rightarrow \sup \left(\sigma \cap N_{\zeta}\right)<f_{\zeta}(\sigma)\right]$. Now use $2.5+$ proof of $[$ Sh 400, $3.5]$.

2.6

We now return to the issue of strong covering (from [Sh:b, Ch.XIII, $§ 1-\S 4]$ ) (better version [Sh:g, Ch.VII]). It influenced the first proof of a bound on $\aleph_{\omega}^{\aleph_{0}}$, and is clearly related to computing $\operatorname{Min}\left\{|S|: S \subseteq \mathscr{S}_{\leqq \kappa}(\lambda)\right.$ is stationary $\}$.

2.7 Lemma. Suppose $\mathbf{W} \subseteq \mathbf{V}$ is a transitive class of $\mathbf{V}$ including all the ordinals and is a model of $Z F C$.

1) For every set $Y \in \mathbf{V}$ of ordinals of cardinality $<\kappa($ in $\mathbf{V})$ there are $Y_{n} \in \mathbf{W} n<$ $\omega$ (so we know only that $\left\langle Y_{n}: n<\omega\right\rangle \in \mathbf{V}$ !), $\mathbf{W} \models$ " $Y_{n}$ a set of $<\kappa$ ordinals" such that $Y \subseteq \bigcup_{n<\omega} Y_{n}$ provided that:

$(*)_{\kappa}(i) \kappa$ is a regular uncountable cardinal in $\mathbf{V}$,

(ii) if $a \in \mathbf{V}$ is a subset of $\operatorname{Reg}^{\mathbf{W}} \backslash \kappa,|a|<\kappa$ and $g \in(\Pi a)^{\mathbf{V}}$ then

$\otimes$ there is a function $h \in \mathbf{W}$ such that $\theta \in \operatorname{Dom}(g) \Rightarrow g(\theta)<h(\theta)<\theta$ (so $\operatorname{Dom}(g) \subseteq \operatorname{Dom}(h))$

or even just

$(*)_{\kappa}^{-}$like $(*)_{\kappa}$ but in (ii) we demand only:

$\otimes^{-} \quad$ there are functions $h_{n} \in \mathbf{W}($ for $n<\omega)$ such that $(\forall \theta \in \operatorname{Dom}(g))\left[\bigvee_{n<\omega} g(\theta)<\right.$ $\left.h_{n}(\theta)<\theta\right]$.

2) For every set $Y \in \mathbf{V}$ of ordinals of cardinality $<\kappa$ (in $\mathbf{V}$ ) there is $Z \in \mathbf{W}$ satisfying $\mathbf{W} \models " Z$ a set of $<\kappa$ ordinals" such that $Y \subseteq Z$ provided that:

$$
(*)_{\kappa}+\aleph_{2}^{\mathbf{V}} \leq \kappa
$$

3) Assume that $\kappa=\aleph_{1}^{\mathbf{V}}$ and $(*)_{\kappa}$ holds and

$\oplus_{0} \mathbf{V} \models$ "A a set of ordinals of power $\kappa$ " $\Rightarrow(\exists B \in \mathbf{W})[A \cap B$ infinite \& $\mathbf{W} \models$ $"|B|<\kappa "]$.

Then the conclusion of part (2) holds. 
(2) holds.

5) Assume

(a) $\left(\kappa^{+}\right)^{\mathbf{V}}=\left(\kappa^{+}\right)^{\mathbf{W}},(*)_{\kappa},(*)_{\kappa^{+}}$;

(b) there is $\left\langle C_{\delta}: \delta \in(\lambda+1) \backslash(\kappa+1), \aleph_{0} \leq c f^{\mathbf{W}} \delta \leq \kappa\right\rangle \in \mathbf{W}$ satisfying $C_{\delta}$ a club of $\delta$ for each $\delta$ such that $\left[\alpha \in \operatorname{acc}\left(C_{\delta}\right) \Rightarrow C_{\alpha}=C_{\delta} \cap \alpha\right]$ and otp $\left(C_{\delta}\right) \leq \kappa$. [nec?]

$\underline{\text { Then }}(\mathbf{W}, \mathbf{V})$ satisfies the $\kappa$-strong covering (see [Sh:g, Ch.VII]) which means:

$\otimes$ for every ordinal $\alpha$ and model $M \in \mathbf{V}$ with universe $\alpha$, with countable vocabulary, there is $N \prec M$ of power $<\kappa, N \cap \kappa$ an ordinal and the universe of $N$ belongs to $\mathbf{W}$.

6) Moreover, in (5)

$\oplus^{+}$in the game where a play last $\kappa$ moves, in the ith move (for $i<\kappa$ ) the first and second players choose $a_{i}, b_{i} \in[\lambda]^{<\kappa}$, respectively preserving $\bigcup_{j<i} b_{j} \subseteq$ $a_{i} \subseteq b_{i}$, the first player has a winning strategy where the first player winning a play means $\left\{\delta<\kappa: \bigcup_{i<\delta} a_{i} \in \mathbf{W}\right\} \in \mathscr{D}_{\kappa}(=$ the clubs filter on $\kappa($ in $\mathbf{V}))$.

Remark. 1) Note that parts (3), (4) hold for $\kappa \geq \aleph_{1}^{\mathbf{V}}$, but this is covered already by part (2).

2) Note that in part (9), $\aleph_{2}^{\mathbf{V}}=\aleph_{2}^{\mathbf{W}}$.

Proof. Should be straightforward (if you read till here). [Originally we say only; for (1) imitate the proof of [Sh 400, 3.5], for (2) - repeat the proof of [Sh 400, 3.5] by doing the induction for $i<\aleph_{1}$, then use part (1). For (3) — instead using part (1) in the end, use the assumption, for (4), (5) imitate the proof of [Sh 400, 3.6].]

For the proof of 1)-5), let $Y$ be a subset of the ordinal $\lambda$, a cardinal of $\mathbf{V}$ (for part (5) $\lambda$ is given), and let $\chi=:\left[\left(2^{\lambda}\right)^{+}\right]^{\mathbf{W}}$. Let $\left(\mathscr{H}(\chi)^{\mathbf{W}}, \in,<_{\chi}^{*}\right) \in \mathbf{W}$.

1) In $\mathbf{V}$ we choose by induction on $n<\omega, N_{n}, \alpha_{n}, h_{n, \ell}, g_{n}$ such that:

(a) $N_{n} \prec\left(\mathscr{H}(\chi)^{\mathbf{W}}, \in,<_{\chi}^{*}\right)$

(b) $\mathbf{V} \models\left\|N_{n}\right\|<\kappa$ and $N_{n} \cap \kappa=\alpha_{n}$;

(c) $Y \subseteq N_{0}$ and $\{\kappa, \lambda\} \in N_{0}$

(d) $N_{n} \prec N_{n+1}$

(e) $h_{n, \ell} \in \mathbf{W}$ is a (partial) function (for $\ell<\omega$ ) and from $\lambda$ to $\lambda$

$(f) g_{n}$ is a function, $\operatorname{Dom}\left(g_{n}\right)=(\lambda \cap \operatorname{Reg} \mathbf{W} \backslash \kappa) \cap N_{n}, g_{n}(\theta)=: \sup \left(N_{n} \cap \theta\right)$

$(g)$ for every $\theta \in \operatorname{Dom}\left(g_{n}\right)$ for some $\ell, g_{n}(\theta)<h_{n, \ell}(\theta)$ 
There is no problem to carry the definition. Let $N=N_{\omega}=: \bigcup_{n<\omega} N_{n}$ and $\alpha_{\omega}^{*}=$ $\bigcup_{n<\omega} \alpha_{n}=N \cap \kappa$. For $\alpha \leq \omega$ let $M_{\alpha}$ be defined as the Skolem Hull in $\left(\mathscr{H}(\chi)^{\mathbf{N}}, \in,<_{\chi}^{*}\right)$ of $\left\{i: i<\alpha_{n}\right\} \cup\left\{h_{n, \ell}: n, \ell<\alpha\right\} \cup\{\kappa, \lambda\}$. Clearly $\left[\alpha<\omega \Rightarrow M_{\alpha} \in \mathbf{W}\right]$, and $\left[\alpha \leq \omega \Rightarrow M_{\alpha} \prec N_{\alpha}\right]$ and $\mathbf{V} \models "\left\|M_{\alpha}\right\|<\kappa "$. Now $M=M_{\omega}=\bigcup_{n<\omega} M_{n} \prec$ $N, M \cap \kappa=\alpha_{\omega}=N \cap \kappa$ and for every $\theta \in\left(\lambda^{+} \cap \operatorname{Reg}^{\mathbf{W}} \backslash \kappa\right) \cap M$ and $n$ for some $m<\omega, \theta \in N_{m} \& m>n$ so for some $\ell<\omega$ we have $\sup \left(N_{n} \cap \theta\right)<h_{m, \ell}(\theta) \in M$ hence $\sup (N \cap \theta)=\sup _{n<\omega}\left(N_{n} \cap \theta\right) \leq \sup (M \cap \theta) \leq \sup (N \cap \theta)$. So by [Sh 400,3.3A], $M \cap \lambda=N \cap \lambda$, so $Y_{n}=: \lambda \cap M_{n}$ for $n<\omega$ are as required.

2)-5) The following will be used in proving during the proof of 2) - 5). Let $\delta(*) \leq \kappa$ be given (i.e. we shall choose it for each part) and we assume $(*)_{\kappa}$. We fix $\left(\mathscr{H}(\chi)^{\mathbf{W}}, \in,<_{\chi}^{*}\right) \in \mathbf{W}$ as in part (1).

In $\mathbf{V}$ we choose by induction on $i<\delta(*), N_{i}, \alpha_{i}, h_{i}, g_{i}$ such that:

(a) $N_{i} \prec\left(\mathscr{H}(\chi)^{\mathbf{W}}, \in,<_{\chi}^{*}\right)$

(b) $\mathbf{V} \models "\left\|N_{i}\right\|<\kappa "$ and $N_{i} \cap \kappa=\alpha_{i}$

(c) $Y \subseteq N_{0},\{\kappa, \lambda\} \subseteq N_{0}$

(d) $N_{i}$ is increasing continuous

(e) $h_{i} \in \mathbf{W}$ is a partial function from $\lambda+1$ to $\lambda+1$

(f) $g_{i}$ is the function with $\operatorname{Dom}\left(g_{i}\right)=\left(\lambda^{+} \cap \operatorname{Reg}{ }^{\mathbf{W}} \backslash \kappa\right) \cap N_{i}$ such that

$$
g_{i}(\theta)=\sup \left(N_{i} \cap \theta\right)
$$

(g) $\theta \in \operatorname{Dom}\left(g_{i}\right) \Rightarrow g_{i}(\theta)<h_{i}(\theta)<\theta$

(h) $h_{i} \in N_{i+1}$.

There is no problem to carry the construction. We let $N=N_{\delta(*)}=\bigcup_{i<\delta(*)} N_{i}$.

Proof of Part (2). Choose $\delta(*)=\aleph_{1}^{\mathbf{V}}$, so under the assumptions of part (2) we have $\delta(*)<\kappa$, hence $\mathbf{V} \models "\|N\|<\kappa$ ". Apply part (1) to $Y=\left\{h_{i}: i<\delta(*)\right\}$ (which is $\subseteq \mathbf{W}$ ), so we can find $Y_{n} \in \mathbf{W}$ (and if you like $Y_{n} \subseteq H=:\{h \in \mathbf{W}: h$ a partial function from $\lambda+1$ to $\lambda+1\}$ ), for $n<\omega$ such that $\mathbf{V} \models$ " $\left|Y_{n}\right|<\kappa$ " and $Y \subseteq \bigcup_{n<\omega} Y_{n}$; (well, $Y$ is not a set of ordinals, but we can code it as one). So for some $n=n(*)$, we hvae $\mathbf{V} \models "\left|Y \cap Y_{n}\right|=\aleph_{1}$ ". Let $M$ be the Skolem Hull in $\left(\mathscr{H}(\chi)^{\mathbf{W}}, \in,<^{*}\right)$ of $\left\{\alpha: \alpha<\bigcup_{i<\delta(*)} \alpha_{i}\right\} \cup Y_{n(*)} \cup\{\kappa, \lambda\}$; so $M \in \mathbf{W}, \mathbf{V} \models "\|M\|<\kappa "$ and so $\mathbf{W} \models "\|M\|<\kappa$ ". Let $M^{\prime}=M \cap N$; so clearly $M^{\prime} \prec N, \bigcup_{i<\delta(*)} \alpha_{i} \subseteq M^{\prime} \cap \kappa \subseteq$ $N \cap \kappa=\bigcup_{i<\delta(*)} \alpha_{i}$. Lastly, if $i<\delta(*)$ and $\theta \in\left(\lambda^{+} \cap \operatorname{Reg} \mathbf{W} \backslash \kappa\right) \cap M^{\prime}$ then for some $j \in(i, \delta(*)), \theta \in N_{j}$ and for some $\epsilon$ we have $j<\epsilon<\delta(*) \quad \& \quad h_{\epsilon} \in Y_{n(*)}$, so 
by [Sh 400, 3.3A] we know $M^{\prime} \cap \lambda=N \cap \lambda$, so $Y \subseteq M^{\prime} \cap \lambda \subseteq M \cap \lambda \in \mathbf{W}$ and $\mathbf{W} \models "|M \cap \lambda| \leq\|M\|<\kappa$ as required. [Saharon: fill details]

Proof of Part (3). As in part (2), we choose $\delta(*)=\aleph_{1}^{\mathbf{V}}$ and get $N_{i}, \alpha_{i}, g_{i}, h_{i}$ (for $i<\delta(*)$ ). By $\oplus_{0}$ applied to $\left\{h_{i}: i<\delta(*)\right\}$ (again translating to a set of ordinals) there is a set $B \in \mathbf{W}$ such that $\mathbf{W} \models "|B|<\kappa "$ and $A \cap B$ infinite, without loss of generality $B \subseteq H$ (see proof of part (2)). So there is a limit ordinal $\zeta<\delta(*)$ such that $\zeta=\sup \left\{i<\zeta: h_{i} \in B\right\}$. Now let $M$ be the Skolem Hull in $\left(\mathscr{H}(\chi)^{\mathbf{W}}, \in,<_{\chi}^{*}\right)$ of $\left\{\alpha: \alpha<\bigcup_{i<\zeta} \alpha_{i}\right\} \cup B \cup\{\kappa, \lambda\}$, and let $M^{\prime}=M \cap N_{\zeta}$ so clearly $M^{\prime} \cap \lambda \subseteq M \cap \lambda \in \mathbf{W},\left|M^{\prime} \cap \lambda\right| \leq|M \cap \lambda| \leq\|M\|<\kappa$ and as above $M^{\prime} \cap \lambda=N_{\zeta} \cap \lambda$, so $N_{\zeta} \cap \lambda=M^{\prime} \cap \lambda \in \mathbf{W}$ but $Y \subseteq N_{\zeta} \cap \lambda=M^{\prime} \cap \lambda \subseteq M \cap \lambda$ and are finished.

Proof of Part (4). We let $\delta(*)=\kappa$ and get $N_{i}, \alpha_{i}, g_{i}, h_{i}$ (for $i<\delta(*)$ ) be as before. Now we apply part (1) with $\kappa^{+}\left(=\left(\kappa^{+}\right)^{\mathbf{V}}=\left(\kappa^{+}\right)^{\mathbf{W}}\right),\left\{h_{i}: i<\kappa\right\}$ here standing for $\kappa$ and $Y$ there, and get $\left\langle Y_{n}: n<\omega\right\rangle$. So for some $n(*)$ the set $\left\{i: g_{i} \in Y_{n(*)}\right\}$ is unbounded in $\kappa$. Let $M$ be the Skolem Hull in $\left(\mathscr{H}(\chi)^{\mathbf{W}}, \in,<_{\chi}^{*}\right)$ of $\{\alpha: \alpha<$ $\left.\bigcup \alpha_{i}\right\} \cup Y_{n(*)} \cup\{\kappa, \lambda\}$. As before $N \subseteq M, M \in \mathbf{W}$ and $\mathbf{W} \models "\|M\|<\kappa^{+} "$. So $i<\delta(*)$

$\mathbf{W} \models "\|M\|=\kappa "$ hence there is a one to one function $f \in W$ from $\{i: i<\kappa\}$ onto $M$, so for some club $E \in \mathbf{V}$ of $\kappa$ (in $\mathbf{V}$ )

$$
i \in E \Rightarrow N_{i} \subseteq \operatorname{Rang}(f\lceil i)
$$

So for each $i \in E, Y \subseteq \operatorname{Rang}(f\lceil i)$ hence we are done.

5 ) So we have already chosen $N_{i}, \alpha_{i}, g_{i}, h_{i}$ for $i<\kappa$. By parts $(2)+(4)$ we can assume without loss of generality that $\operatorname{Dom}\left(h_{i}\right)$ has cardinality $<\kappa$. Let $N_{\kappa}=$ $\bigcup_{i<\kappa} N_{i}$, by part $(1)$ there is a sequence $\left\langle Y_{n}: n<\omega\right\rangle$ satisfying $Y_{n} \in \mathbf{W}$ such that:

$$
\begin{array}{r}
\left\{h_{i}: i<\kappa\right\} \subseteq \bigcup_{n<\omega} Y_{n} \subseteq H=\{f \in \mathbf{W}: f \text { a partial function from } \\
\left.\qquad(\lambda+1) \cap \operatorname{Reg}^{\mathbf{W}} \backslash \kappa \text { to } \lambda+1\right\}
\end{array}
$$

and for each $n, \mathbf{W} \models "\left|Y_{n}\right|<\kappa^{+"}$. So for some $n(*),\left\{i<\kappa: h_{i} \in Y_{n(*)}\right\}$ is unbounded in $\kappa$. So in $\mathbf{W}$ there is a list $\left\langle f_{i}: i<\kappa\right\rangle$ of $Y_{n(*)}$. In $\mathbf{V}$, for each $i<\kappa$ let $j_{i}<\kappa$ be minimal such that:

(a) $h_{i} \in\left\{f_{\zeta}: \zeta<j_{i}\right\}$

(b) if for some $\zeta<\kappa, f_{i} \leq h_{\zeta}$ (i.e. $\operatorname{Dom}\left(f_{i}\right) \subseteq \operatorname{Dom}\left(h_{\zeta}\right)$ and $\left(\forall \theta \in \operatorname{Dom}\left(f_{i}\right)\right)\left[f_{i}(\theta) \leq\right.$ $\left.h_{\zeta}(\theta)\right]$ then there is such $\zeta<j_{i}$

(c) $j_{i} \geq i+1$.

Let $E=\left\{\zeta<\kappa\right.$ : for every $i<\zeta, j_{i}<\zeta$, and $\zeta$ is a limit ordinal $\}$. Now for each $\zeta \in E$ note that

$(*)_{1}(\alpha)\left\{h_{i}: i<\zeta\right\} \subseteq\left\{f_{i}: i<\zeta\right\} ;$

( $\beta) \bigwedge\left[\bigvee f_{i} \leq h_{j} \leftrightarrow \bigvee f_{i} \leq h_{j}\right]$ 
As $\left[i<j \Rightarrow h_{i}<h_{j}\right]$ clearly we get:

$$
(\beta)^{\prime} \bigwedge_{i<\zeta j<\zeta}\left[\bigvee_{i<} f_{i} \leq h_{j} \Leftrightarrow f_{i} \leq h_{\zeta}\right]
$$

Define for $\zeta \leq \kappa$ a function $f_{\zeta}^{*}$ as follows:

$(*)_{2} \operatorname{Dom}\left(f_{\zeta}^{*}\right)=\bigcup\left\{\operatorname{Dom}\left(f_{i}\right): i<\zeta\right.$ and $\left.f_{i} \leqq h_{\zeta}\right\}$ $f_{\zeta}^{*}(\theta)=\sup \left\{f_{i}(\theta): i<\zeta\right.$ and $\left.f_{i} \leqq h_{\zeta}\right\}$.

So clearly

$(*)_{3}$ for $\zeta \in E, \operatorname{Dom}\left(f_{\zeta}^{*}\right)=\cup\left\{\operatorname{Dom}\left(h_{i}\right): i<\zeta\right\}, f_{\zeta}^{*}(\theta)=\sup \left\{h_{i}(\theta): i<\zeta\right\}$ hence $\operatorname{Dom}\left(f_{\zeta}^{*}\right)=N_{\zeta^{\prime}} \cap(\lambda+1) \cap \operatorname{Reg}^{\mathbf{W}} \backslash(\kappa+1)$

$(*)_{4} f_{\zeta}^{*} \in \mathbf{W}$, and even for $\left\langle f_{\zeta}^{*}: \zeta<\kappa\right.$ limit $\rangle \in \mathbf{W}$. Let us define for $\zeta \leq \kappa$ a function $g_{\zeta}^{*}$ :

$$
\begin{gathered}
\operatorname{Dom}\left(g_{\zeta}^{*}\right)=\bigcup_{i<\zeta} \operatorname{Dom}\left(h_{i}\right) \subseteq(\lambda+1) \cap \operatorname{Reg}^{\mathbf{W}} \backslash \kappa, \\
g_{\zeta}^{*}(\theta)=\bigcup_{i<\zeta} g_{i}(\theta) .
\end{gathered}
$$

Clearly for $\zeta$ limit:

$$
(*)_{5} \operatorname{Dom}\left(g_{\zeta}^{*}\right)=(\lambda+1) \cap \operatorname{Reg}^{\mathbf{W}} \cap N_{\zeta} \backslash \kappa
$$

and if $\zeta \in E$

$$
\begin{aligned}
\theta \in \operatorname{Dom}\left(g_{\zeta}^{*}\right) \Rightarrow g_{\zeta}^{*}(\theta) & =\bigcup\left\{g_{i}(\theta): i<\zeta, \theta \in N_{i}\right\}=\bigcup\left\{h_{i}(\theta): i<\zeta, \theta \in N_{i}\right\} \\
& =\bigcup\left\{f_{i}(\theta): i<\zeta \text { and } f_{i} \leq h_{\zeta}\right\}=f_{\zeta}^{*}(\theta) .
\end{aligned}
$$

So for $\zeta \in E$ we have $g_{\zeta}^{*} \subseteq f_{\zeta}^{*}$ but by $(*)_{3}+(*)_{5}$ they have the same domain hence $g_{\zeta}^{*}=f_{\zeta}^{*}$.

For every $\theta \in N_{\kappa} \cap\left((\lambda+1) \cap \operatorname{Reg}^{\mathbf{W}} \backslash(\kappa+1)\right), g_{\kappa}(\theta)$ is an ordinal $\in(\kappa, \theta) \subseteq(\kappa, \lambda)$ of cofinality $\kappa$, so $C_{g_{\kappa}(\theta)}$ is a set of ordinals of order type $\kappa$; let $C_{g_{\kappa}(\theta)}=\left\{\alpha_{\zeta}^{\theta}\right.$ : $\zeta<\kappa\}$, (increasing); it is strictly increasing continuous and has limit $g_{\kappa}(\theta)$; also $\left\langle g_{\zeta}^{*}(\theta): \zeta<\kappa\right\rangle$ is also strictly increasing continuous with limit $g_{\kappa}^{*}(\theta)$. Clearly $E_{\theta}=\left\{\zeta<\kappa: \zeta\right.$ limit ordinal and $\alpha_{\zeta}^{\theta}=g_{\zeta}^{*}(\theta)$ (so $\left.\left.\theta \in N_{\zeta}\right)\right\}$ is a club of $\kappa$ (in $V$; as $V \models " \kappa$ regular uncountable"). So for $\zeta \in E_{\theta}, C_{\alpha_{\zeta}^{\theta}}=C_{g_{\kappa}(\theta)} \cap \alpha_{\zeta}^{\theta} \subseteq N_{\zeta+1}$, hence $\left[\zeta \in \operatorname{acc} E_{\theta} \Rightarrow C_{g_{\kappa}(\theta)} \cap \alpha_{\zeta}^{\theta} \subseteq N_{\zeta}\right]$. Let $E^{*}=\left\{\zeta \in E:(\forall i<\zeta)\left(\forall \theta \in N_{i}\right)[\theta \in\right.$ $\left.(\lambda+1) \cap \operatorname{Reg}^{W} \backslash(\kappa+1) \Rightarrow \zeta \in \operatorname{acc} E_{\theta}\right\}$. So for $\zeta \in E^{*}, \zeta$ is a limit ordinal and:

$$
\theta \in N_{\zeta} \cap\left((\lambda+1) \cap \operatorname{Reg}^{W} \backslash(\kappa+1)\right) \Rightarrow C_{f_{\zeta}^{*}(\theta)}=C_{g_{\zeta}^{*}(\theta)}
$$


Now we shall show that for $\zeta \in E^{*}, N_{\zeta} \cap \lambda \in W$. For $\zeta \in E^{*}$ we define by induction on $n<\omega, M_{\zeta}^{n}$ :

$$
M_{\zeta}^{0} \text { is the Skolem Hull in }\left(\mathscr{H}(\chi)^{\mathbf{W}}, \in,<^{*}\right) \text { of }\left\{\alpha: \alpha<\alpha_{\zeta}\right\} \text {. }
$$

Let $M_{\zeta}^{n+1}$ be the Skolem Hull in $\left(\mathscr{H}(\chi)^{\mathbf{W}}, \in,<_{\chi}^{*}\right)$ of

$$
M_{\zeta}^{n} \cup \bigcup\left\{C_{f_{\zeta}^{*}(\theta)}: \theta \in(\lambda+1) \cap \operatorname{Reg}^{\mathbf{W}} \backslash(\kappa+1) \text { and } \theta \in M_{\zeta}^{n} \cap \operatorname{Dom} f_{\zeta}^{*}\right\}
$$

Let $M_{\zeta}=\bigcup_{n<\omega} M_{\zeta}^{n}$, clearly $M_{\zeta} \in \mathbf{W}\left(\right.$ as $\left.f_{\zeta}^{*} \in \mathbf{W}\right)$ we can prove by induction on $n$ that $M_{\zeta}^{n} \subseteq N_{\zeta}$, hence: $M_{\zeta} \subseteq N_{\zeta}$. Also $\alpha_{\zeta} \subseteq M_{\zeta} \cap \kappa \subseteq N_{\zeta} \cap \kappa=\alpha_{\zeta}$, and:

$\theta \in M_{\zeta} \cap\left((\lambda+1) \cap \operatorname{Reg}^{W} \backslash(\kappa+1)\right) \Rightarrow C_{g_{\zeta}^{*}(\theta)}$ is an unbounded of subsets of $\operatorname{both} N_{\zeta} \cap \theta, M_{\zeta} \cap \theta$.

So by $[$ Sh $400,5.1 \mathrm{~A}(1)]$ we get $N_{\zeta} \cap \lambda=M_{\zeta} \cap \lambda$. Alternatively, let for $\zeta \in E, M_{\zeta}^{*}$ is the Skolem hull of $\alpha_{\zeta} \cup\left\{f_{i}^{*}: i<\zeta\right\}$ in $\left(\mathscr{H}(\chi)^{\mathbf{W}}, \in,<_{\chi}^{*}\right)$; so clearly $M_{\zeta}^{*} \subseteq N_{\zeta}$ and again by $[$ Sh $400,5.1 \mathrm{~A}]$ we have $M_{\zeta}^{*} \cap \lambda=N_{\zeta} \cap \lambda$.

6) The winning strategy for the second player is to choose "on the side" also $N_{i}, \alpha_{i}, h_{i}, g_{i}$ as in the common part of the proof of parts (2)-(5) and guaranteeing: $a_{i}$ include $\bigcup_{j<i}\left(N_{j} \cap \lambda\right) \cup \bigcup_{j<i} b_{j}, N_{i+1}$ include $b_{i}$ and $N_{i} \cap \lambda$ is the universe of an elementary submodel of $\left(\mathscr{H}(\chi)^{\mathbf{W}}, \in,<_{\chi}^{*}\right) \in \mathbf{W} . \quad \square_{2.7}$

2.8 Remark. 1) We can put $\lambda$ as a parameter of the Lemma 2.7, then in $(*)_{\kappa}, a \subseteq$ $(\lambda+1) \cap \operatorname{Reg}^{\mathbf{W}} \backslash \kappa$, etc., (so we may write $\left.(*)_{\kappa}\right)$ and $Y \subseteq \lambda$ (in parts (1)-(4)) and $\alpha \leq \lambda$ (in $\otimes$ of part (5)).

2) Note that $(*)_{\kappa}^{\lambda}$ follows easily from the relevant covering property in [Sh:g, Ch.VII]:

$(*)$ if $a \in \mathbf{V}, a \subseteq \lambda, \mathbf{V} \models "|a|<\kappa "$ then for some $b \in \mathbf{W}, a \subseteq b, \mathbf{W} \models "|b|<\kappa "$. 


\section{$\S 3$ PCF Inaccessibility and Characterizing the Existence of Non <-Decreasing Sequences (for a Topological Problem)}

3.1 Definition. 1) We say $\lambda$ is $(\mu, \theta, \sigma)$-inaccessible if $\lambda>\mu \geq \theta>\sigma$ and for any $\mathfrak{a} \subseteq$ Reg we have: if $|\mathfrak{a}|<\theta, \operatorname{Min}(\mathfrak{a})>\mu$ and $\mathfrak{a} \subseteq \lambda$, even $\sup (\mathfrak{a})<\lambda$ and $I$ is a $\sigma$-complete ideal on $\mathfrak{a}$, then $\lambda \not \leq \operatorname{tcf}(\Pi \mathfrak{a} / I)$ (when tcf is well defined).

2) If we write $*$ instead of $\mu$ we mean "for some $\mu, \theta \leq \mu<\lambda$ ".

3) If we omit $\sigma$ we mean $\sigma=\aleph_{0}$.

4) "accessible" is just the negation of "inaccessible".

We now rephrase various old results.

3.2 Claim. 1) For $\lambda$ regular, in the definition, "and $\sigma$-complete $I, \lambda \not \operatorname{tcf}(\Pi \mathfrak{a} / I)$ " can be replaced by " $\lambda \notin \operatorname{pcf}_{\sigma \text {-complete }}(\mathfrak{a})$ " and also by " $\lambda \neq \operatorname{tcf}(\Pi \mathfrak{a} / I)$ for any $\mathfrak{a} \subseteq$ Reg $\cap(\mu, \lambda),|\mathfrak{a}|<\theta, I$ being $\sigma$-complete"; also if $\operatorname{cf}(\lambda) \notin[\sigma, \theta)$ then $" \sup (\mathfrak{a})<\lambda "$ is not necessary just " $\lambda \notin \mathfrak{a}$ ".

2) Assume $\lambda>\mu \geq \theta>\sigma$ and $\operatorname{cf}(\lambda) \geq \sigma$. Then $\lambda$ is $(\mu, \theta, \sigma)$-inaccessible iff $\left[\lambda^{\prime} \in(\mu, \lambda) \& \sigma \leq \operatorname{cf}\left(\lambda^{\prime}\right)<\theta \Rightarrow \operatorname{pp}_{\Gamma(\theta, \sigma)}\left(\lambda^{\prime}\right)<\lambda\right]$.

3) If $\lambda=\operatorname{cf}(\lambda)>\mu \geq \theta=\operatorname{cf}(\theta)>\sigma, \lambda$ is $(\mu, \theta, \sigma)$-accessible then there is a set $\mathfrak{a} \subseteq \operatorname{Reg} \cap(\mu, \lambda)$ of $(\mu, \theta, \sigma)$-inaccessible cardinals each $>\mu$, $\mathfrak{a}$ of cardinality $<\theta$ such that $\lambda \in \operatorname{pcf}_{\sigma \text {-complete }}(\mathfrak{a})$.

4) If $\lambda=\operatorname{cf}(\lambda) \geq \kappa>\mu \geq \theta>\sigma=\operatorname{cf}(\sigma)$, and $(\exists \mathfrak{a})[\mathfrak{a} \in \operatorname{Reg} \cap(\mu, \kappa) \&|\mathfrak{a}|<\theta$ \& $\left.\lambda \in \operatorname{pcf}_{\sigma \text {-complete }}(\mathfrak{a})\right]$ then there is a set $\mathfrak{a}$ of $(\mu, \theta, \sigma)$-inaccessible cardinals $\in(\mu, \kappa)$ with $|\mathfrak{a}|<\theta$ such that $\lambda=\max \operatorname{pcf}(\mathfrak{a})$, and $\lambda \in \operatorname{pcf}_{\sigma \text {-complete }}(\mathfrak{a})$. If $\kappa$ is $(\mu, \theta, \sigma)$ inaccessible then necessarily $\sup (\mathfrak{a})=\kappa, J_{\mathfrak{a}}^{b d} \subseteq J_{<\lambda}[\mathfrak{a}]$. If $\sigma=\aleph_{0}$, also there is a tree of cardinality $\leqq \kappa$ and $\geq \lambda((c f \kappa)$-branches if $\kappa$ is $(\mu, \theta, \sigma)$-inaccessible $)$.

5) If $\lambda=\max p c f(\mathfrak{a}), \kappa=|\mathfrak{a}| \leq \mu<\operatorname{Min}(\mathfrak{a})$, each $\theta \in \mathfrak{a}$ is $\left(\mu, \kappa^{+}, 2\right)$-inaccessible then there is a tree of cardinality $\sup (\mathfrak{a})$ and $\geq \lambda \mathrm{cf}(\mathrm{otp})$-branches. If we have $\kappa$ pairwise disjoint subsets of $\mathfrak{a}$ not in $J_{<\lambda}[\mathfrak{a}], 2^{\kappa} \geq \sup (\mathfrak{a})$ or on each $\theta \in \mathfrak{a}$ there is an entangled linear order then there is an entangled linear order of cardinality $\lambda$.

6) If $\mu<\lambda<p p^{+}(\mu)$, then there is a tree with $\leq \mu$ nodes and $\geq \lambda$ branches. If $\mu$ $i s\left(*,(c f \mu)^{+}, 2\right)$-inaccessible we can demand " $\geq \lambda($ cf $\mu)$-branches".

Proof. 1) Easy (using pcf analysis and [Sh 355, §1]).

2) Easy, too (use $[$ Sh 355, 2.4]).

3) Prove by induction on $\lambda$ using [Sh 345a, 1.10] (so in [Sh 345a, 1.12] we can replace pcf by $\left.\mathrm{pcf}_{\sigma \text {-complete }}\right)$.

4) Similar to (3).

5) By $[\mathrm{Sh} 355, \S 4]$.

6) Easy, too.

We state some variants of [Sh 400, $§ 2, \S 4]$; specifically combining [Sh 400, 2.4,4.2]:

3.3 Claim. Suppose: 
(ii) for $\zeta$ limit, $\lambda_{\zeta}=\left(\bigcup_{\xi<\zeta} \lambda_{\xi}\right)^{+}$

(iii) $\lambda_{\zeta} \in \operatorname{pcf}\left(\mathfrak{a}_{\zeta}\right)$

(iv) $\mathfrak{a}_{\zeta} \subseteq \operatorname{Reg} \cap\left(\sigma^{+}, \lambda_{0}\right)$ and $\left|\mathfrak{a}_{\zeta}\right|<\sigma, \sigma$ regular.

Then $\zeta(*)<\sigma^{+3}$.

Similarly combining [Sh 400, 2.4,4.2]

3.4 Claim. Suppose:

(i) $\left\langle\lambda_{\zeta}: \zeta<\zeta(*)\right\rangle$ is a strictly increasing sequence of regular cardinals $>\kappa$

(ii) for $\zeta$ limit, $\lambda_{\zeta}=\left(\bigcup_{\xi<\zeta} \lambda_{\xi}\right)^{+}$sn

(iii) $\lambda_{\zeta} \in p c f_{\sigma \text {-complete }}\left(a_{\zeta}\right)$ where $\sigma$ is regular

(iv) $\mathfrak{a}_{\zeta} \subseteq \operatorname{Reg} \cap\left(\kappa^{+}, \lambda_{0}\right),\left|\mathfrak{a}_{\zeta}\right|<\kappa, \kappa$ regular

(v) $\mathfrak{a}_{\zeta} \subseteq \mathfrak{b}$ and if $\left\langle\mu_{\zeta}: \zeta \leqq \kappa^{+2}\right\rangle$ is strictly increasing sequence of regular $\mathfrak{a} \subseteq \mathfrak{b},|\mathfrak{a}|<\kappa,\left\{\mu_{\zeta}: \zeta<\kappa^{+2}\right\} \subseteq \operatorname{pcf}(\mathfrak{a}), \mu_{\kappa^{+2}}=\max \operatorname{pcf}\left\{\mu_{\zeta}: \zeta<\kappa^{+2}\right\}$ and $\left(\lambda_{0}+\sum_{\zeta<\kappa^{+2}} \mu_{\zeta}\right)^{+}<\mu_{\kappa^{+2}}<\bigcup_{\zeta<\xi}\left\{\lambda_{\zeta}: \zeta<\kappa^{+3}\right\}$, then there are $\mu_{\zeta}^{\prime}<\mu_{\zeta}$ regular, as for $\zeta \leqq \kappa^{+2}$ and $\mathfrak{a}^{\prime} \subseteq \mathfrak{b}$ with the same properties and $\Sigma\left\{\mu_{\zeta}: \zeta<\right.$ $\left.\kappa^{+2}\right\}=\Sigma\left\{\mu_{\zeta}^{\prime}: \zeta<\kappa^{+2}\right\}$.

$\underline{\text { Then }} \zeta(*)<\operatorname{Max}\left\{\kappa^{+3}, \operatorname{cov}\left(\bigcup_{\zeta<\xi} \mathfrak{a}_{\zeta}, \kappa, \kappa, \sigma\right)^{+}\right\}$.

3.5 Claim. The following is impossible:

(i) $\sigma<\kappa<\theta<\mu$ are regular, $\kappa^{+}<\theta$

(ii) $\left\langle\lambda_{\zeta}: \zeta<\mu\right\rangle$ is a strictly increasing sequence of regular cardinals $>\mu$

(iii) $S=\left\{\epsilon<\mu: \operatorname{cf}(\epsilon)=\theta\right.$ and for some club $C$ of $\epsilon$, sup $\operatorname{pcf}_{\sigma \text {-complete }}\left\{\lambda_{\zeta}: \zeta \in\right.$ $\left.C\}<\sum_{\zeta<\mu} \lambda_{\zeta}\right\}$ is stationary;

(iv) (a) if $\delta<\mu, c f(\delta)=\kappa$ then for every club $C$ of $\delta$, there is $\alpha \in C$ such that

$$
\sup \operatorname{pcf}_{\sigma \text {-complete }}\left\{\lambda_{\zeta}: \zeta \in \alpha \cap C\right\} \geq \bigcup_{\zeta \in C} \lambda_{\zeta}
$$

or

(b) $\quad \lambda_{\zeta} \in p c f_{\sigma \text {-complete }}\left(\mathfrak{a}_{\zeta}\right),\left|\mathfrak{a}_{\zeta}\right|<\kappa$ and $\mu>\operatorname{cov}\left(\bigcup_{\zeta<\mu} \mathfrak{a}_{\zeta}, \kappa, \kappa, \sigma\right)$. 
3.6 Claim. Assume $|\mathfrak{a}|<\operatorname{Min}(\mathfrak{a})$, then

$$
\mathrm{cf}_{\leq|\mathfrak{a}|}(\Pi \operatorname{pcf}(\mathfrak{a})) \leq \max \operatorname{pcf}(\mathfrak{a})
$$

Proof. More is proved in [Sh 371, §3].

The following answers a question of Gerlits, Hajnal and Szentmiklossy in [GHS]. They dealt with " $\kappa$-good topological spaces $X$ " (i.e. every subset is the union of $\leq \kappa$ compact sets) and "weakly $\kappa$-good spaces" (every $Y \subseteq X$ of cardinality $>\kappa$ contains a compact subset of cardinality $>\kappa)$. [GHS] has the easy implication.

We return to this in $[$ Sh $513, \S 6]$.

3.7 Theorem. The following conditions on $\kappa<\sigma<\theta$ are equivalent: ( $\kappa$ is an infinite cardinal, $\sigma$ and $\theta$ are ordinals)

$(A)_{\kappa, \sigma, \theta}$ there are functions $f_{\alpha}: \kappa \rightarrow \sigma$ for $\alpha<\theta$ such that:

$$
\alpha<\beta \Rightarrow \bigvee_{i<\kappa} f_{\alpha}(i)<f_{\beta}(i)
$$

$(B)_{\kappa, \sigma, \theta} 2^{\kappa} \geq|\theta|$ or for every regular $\mu_{1} \leq \theta$ for some singular cardinal $\lambda^{*} \leq \sigma$ we have:

$$
\operatorname{cf}\left(\lambda^{*}\right) \leq \kappa, \lambda^{*}>2^{\kappa}, \mathrm{pp}^{+}\left(\lambda^{*}\right)>\mu_{1}
$$

Proof. First note

3.8 Observation. Let $\kappa<\sigma, \kappa$ an infinite cardinal, $\sigma, \theta$ are ordinals. If for every regular $\theta_{1}, \sigma \leq \theta_{1}<\theta$ the statement $(A)_{\kappa, \sigma, \theta_{1}}$ holds and $\theta$ is singular (e.g. $\theta>|\theta|$ ), then $(A)_{\kappa, \sigma, \theta}$ holds.

Proof. We prove this by induction on $\theta$; if $\theta \leq \sigma-$ trivial: use the constant functions. As $\theta$ is singular $\theta=\sum_{\alpha<\theta(*)} \theta_{\alpha}$ where $\theta(*)<\theta, \theta_{\alpha}<\theta, \theta_{\alpha}$ increasing continuous, $\theta_{0}=0$. By the assumption for each $\alpha<\theta(*)$, there is a sequence $\left\langle f_{i}^{\alpha}: i<\theta_{\alpha}\right\rangle$ as required in $(A)_{\kappa, \sigma, \theta_{\alpha}}$, [why? if $\theta_{\alpha}$ is singular by the induction hypothesis, if $\theta_{\alpha}$ is regular by an assumption of 3.8]. Similarly there is $\left\langle f_{i}: i<\theta(*)\right\rangle$ exemplifying $(A)_{\kappa, \sigma, \theta(*)}$.

For $i<\theta$ let $i=\sum_{\beta<\alpha(i)} \theta_{\beta}+j(i), j(i)<\theta_{\alpha(i)}, \alpha(i)<\theta(*)$ and let $g_{i}: \kappa \rightarrow \lambda$ be $g_{i}(2 \zeta)=f_{\alpha(i)}(\zeta), g_{i}(2 \zeta+1)=f_{j(i)}^{\alpha(i)}(\zeta)$ 
$\underline{(B) \Rightarrow(A)}$

First Case: $2^{\kappa} \geq|\theta|$.

Let $\left\{A_{\alpha}: \alpha<\theta\right\}$ be a family of $|\theta|$ distinct subsets of $\kappa$, let $A_{\alpha}^{\prime}=\{2 i: i \in$ $\left.A_{\alpha}\right\} \cup\left\{2 i+1: i \notin A_{\alpha}, i<\kappa\right\}$ and let $f_{\alpha}: \theta \rightarrow\{0,1\} \subseteq \lambda$ be

$$
f_{\alpha}(i)= \begin{cases}0 & \text { if } i \in A_{\alpha}^{\prime} \\ 1 & \text { if } i \notin A_{\alpha}^{\prime}\end{cases}
$$

Second Case: $\lambda^{*} \leqq \sigma, \operatorname{cf}\left(\lambda^{*}\right) \leq \kappa, \lambda^{*}>2^{\kappa}, \mathrm{pp}^{+}\left(\lambda^{*}\right)>\theta, \theta$ a regular cardinal.

So there are regular cardinals $\lambda_{i}<\lambda^{*}$ for $i<\kappa$ (such that $\lambda_{i}>\kappa$ ) and an ideal $J$ on $\kappa, \kappa \notin J$ such that $\prod_{i<\kappa} \lambda_{i} / J$ has true cofinality $\theta_{1} \geq \theta$. So there is a sequence $\left\langle f_{\alpha}: \alpha<\theta_{1}\right\rangle, f_{\alpha} \in \prod_{i<\kappa} \lambda_{i}$ and $\alpha<\beta \Rightarrow f_{\alpha}<f_{\beta} \bmod J$. Now $\left\langle f_{\alpha}: \alpha<\theta\right\rangle$ is a sequence as required.

By 3.8 those two cases suffice.

$\underline{(A) \Rightarrow(B)}$

Let $\left\langle f_{\alpha}: \alpha<\theta\right\rangle$ be as in (A).

We can assume that $(B)$ fails, $\sigma$ minimal for which this occurs (for a given $\kappa$ for some $\theta$ ) and $\theta$ minimal for the given $\kappa$ and $\sigma$. So $\theta \geq\left(2^{\kappa}\right)^{+}$. By Observation 3.8, $\theta$ is a regular cardinal. So $2^{\kappa}<\theta$ (hence $2^{\kappa}<\sigma$ ) and $\left[a \subseteq \operatorname{Reg} \cap \sigma^{+} \backslash \kappa^{+} \&|a| \leq\right.$ $\kappa \Rightarrow \max \operatorname{pcf} a<\theta$ ], and $\sigma<\theta$.

As $\theta$ is a regular cardinal necessarily $\operatorname{cf}(\sigma) \leq \kappa$ (otherwise for some $\sigma_{1}<\sigma$ the set $\left\{\alpha<\theta: \operatorname{Rang}\left(f_{\alpha}\right) \subseteq \sigma_{1}\right\}$ is unbounded in $\theta$, contradicting the minimality of $\sigma$ ). Also $\sigma$ is a limit ordinal as $2^{\kappa}<\theta=\operatorname{cf}(\theta)$ (as if $\sigma=\beta+1$, for some $A \subseteq \kappa, B=\left\{\alpha: \bigwedge_{i<\kappa}\left[i \in A \Leftrightarrow f_{\alpha}(i)=\beta\right]\right\}$ has cardinality $\theta$, so $\left\{f_{\alpha}\lceil(\kappa \backslash A): \alpha \in B\}\right.$ essentially contradicts the minimality of $\sigma$ ).

Let $\chi$ be regular large enough. We choose by induction on $i<\left(2^{\kappa}\right)^{+}$, a model $N_{i}$ such that:

$$
\begin{aligned}
& N_{i} \prec\left(\mathscr{H}(\chi), \in,<_{\chi}^{*}\right) \\
& \left\|N_{i}\right\|=2^{\kappa} \\
& 2^{\kappa} \subseteq N_{0} \\
& \kappa, \sigma, \theta \in N_{0},\left\langle f_{\alpha}: \alpha<\theta\right\rangle \in N_{0} \\
& i<j \Rightarrow N_{i} \prec N_{j} \\
& \left\langle N_{j}: j \leq i\right\rangle \in N_{i+1} \\
& N_{i} \text { increasing continuous. }
\end{aligned}
$$

Let $\delta_{i}=: \sup \left(\theta \cap N_{i}\right)$ so $\left\langle\delta_{i}: i<\left(2^{\kappa}\right)^{+}\right\rangle$is strictly increasing continuous (as $\theta$ is regular, $\theta>\sigma$ and $\sigma>2^{\kappa}$, necessarily $\left.\delta_{i}<\theta\right)$. We define for $i<\left(2^{\kappa}\right)^{+}$, a function $g_{i} \in{ }^{\kappa} \sigma$ by

$$
g_{i}(\zeta)=\operatorname{Min}\left(N_{i} \cap \sigma \backslash f_{\delta_{i}}(\zeta)\right)
$$

(it is well defined as $\sigma \in N_{0} \subseteq N_{i}$ and $N \cap \sigma$ is unbounded in $\sigma$ as cf $\sigma \leq \kappa$ ).

Now $i<\left(2^{\kappa}\right)^{+}, \operatorname{cf}(i)=\kappa^{+}$implies $N_{i}=\bigcup_{j<i} N_{j}$ and Rang $g_{i} \subseteq \bigcup_{j<i} N_{j}$ hence $\bigvee\left[\operatorname{Rang}\left(g_{i}\right) \subseteq N_{j}\right]$; but every subset of $N_{j}$ of cardinality $\leq \kappa$ belongs to $N_{j+1}$, 
hence $g_{i} \in \bigcup_{j<i} N_{j}$. So by Fodor Lemma for some stationary subset $S$ of $\left\{i<\left(2^{\kappa}\right)^{+}\right.$: $\left.\operatorname{cf}(i)=\kappa^{+}\right\}$and some $g^{*}: \kappa \rightarrow \sigma$ and some $A \subseteq \kappa$ and some $i(*)<\left(2^{\kappa}\right)^{+}$we have: $\left[i \in S \Rightarrow g_{i}=g^{*}\right],(\forall i \in S)(\forall \zeta<\kappa)\left[f_{\delta_{i}}(\zeta)=g^{*}(\zeta) \Leftrightarrow \zeta \notin A\right]$ and $g^{*} \in N_{i(*)}$; note $A \in N_{0} \subseteq N_{i(*)}$ as $A \subseteq \kappa$.

Clearly $i \in S \quad \& \quad \zeta \in A \Rightarrow \operatorname{cf}\left[g^{*}(\zeta)\right]>2^{\kappa}$ (otherwise $g^{*}(\zeta)=\sup \left(N_{i} \cap g^{*}(\zeta)\right.$ ) (as $\left.N_{i} \prec\left(\mathscr{H}(\chi), \in,<_{\chi}^{*}\right), 2^{\kappa}+1 \subseteq N_{0} \subseteq N_{i}\right)$ and easy contradiction). Also, as the $f_{\alpha}$ 's are pairwise distinct, clearly $A \neq \emptyset$.

Question: What is $\operatorname{cf}\left[\prod_{\zeta \in A} \operatorname{cf}\left[g^{*}(\zeta)\right]\right]$ ?

(I.e. cofinality of the partial ordered set).

By $\left[\right.$ Sh 355, 3.1] it is $\max \operatorname{pcf}\left\{\operatorname{cf}\left[g^{*}(\zeta)\right]: \zeta \in A\right\}$, which by an assumption is $<\theta$, so there is a family $G \subseteq \prod_{\zeta \in A} g^{*}(\zeta)$ of cardinality $<\theta$ such that $\left(\forall f \in \prod_{\zeta \in A} g^{*}(\zeta)\right)(\exists g \in$ $G)[f<g]$. As the parameters in the demands on $G$ belongs to $N_{i(*)}$, without loss of generality $G \in N_{i(*)}$.

Now we can define a partial function $H$ from the family $G$ to $\theta$ :

if $g \in G$ and for some $\alpha$ the condition $(*)$ below holds then $H(g)$ is such an ordinal

if $g \in G$ and for no $\alpha$ the condition $(*)$ below holds then $H(g)$ is not defined where

(*) $\alpha<\theta, f_{\alpha} \uparrow(\kappa \backslash A)=g^{*} \uparrow(\kappa \backslash A)$ and $g=g \uparrow A \leq f_{\alpha} \uparrow A \leq g^{*} \uparrow A$.

Now we can choose an ordinal $j(*)$ such that

$$
i(*)<j(*)<\left(2^{\kappa}\right)^{+}, \quad j(*) \in S
$$

(possible as $S$ is a stationary subset of $\left(2^{\kappa}\right)^{+}$).

We know that there is a function $h \in G$ such that $f_{\delta_{j(*)}} \uparrow A<h$.

Question: Is $H(h)$ well defined?

Possibility A: The answer is yes.

Then $H(h)<\cup\{H(g)+1: g \in(\operatorname{Dom}(H)) \subseteq G\}$. This union is an ordinal $<\theta$ (as $|G|<\theta$ and $\operatorname{Rang}(H) \subseteq \theta$ and $\theta$ is regular); also this union belongs to $N_{i(*)}$ (as $\left.G, H \in N_{i(*)}\right)$, hence the union is an ordinal $<\delta_{i(*)}<\delta_{j(*)}$. So $H(h)<\delta_{j(*)}$.

But (by the choice of $h$ for the first inequality, and definition of $H(h)$ for the second inequality)

$$
\otimes_{1} f_{\delta_{j(*)}}\left\lceil A \leq g_{j(*)} \uparrow A<h \uparrow A \leq f_{H(h)} \uparrow A\right.
$$

and (by the definition of $H$ for the first equality, choice of $g^{*}$ and $j(*) \in S$ for the second): 
Now $\otimes_{1}, \otimes_{2}$ together implies $f_{\delta_{j(*)}} \leq f_{H(h)}$, but as said earlier $H(h)<\delta_{j(*)}<\theta$, together they contradict the choice of $\left\langle f_{\alpha}: \alpha<\theta\right\rangle$.

Possibility $B$ : The answer is no.

So $H(h)$ is not well defined and without loss of generality $h \in N_{j(*)+1}$ (as all parameters in the requirements on it are in $\left.N_{j(*)+1}\right)$. Choose $j \in S, j>j(*)$; as $H(h)$ is not well defined, no $\alpha<\theta$ satisfies the requirements in $(*)$. But of the three demands on $\alpha, \delta_{j}$ trivially satisfy two and a half: " $\alpha<\theta, f_{\alpha}\left\lceil(\kappa \backslash A)=g^{*}\lceil(\kappa \backslash A)\right.$ and $f_{\alpha}\left\lceil A \leq g^{*} \uparrow A^{\prime \prime}\right.$; so the remaining one should fail, i.e. $\neg\left[h\left\lceil A \leq f_{\delta_{j}}\lceil A]\right.\right.$. So for some $\zeta \in A$ we have $h(\zeta)>f_{\delta_{j}}(\zeta)$; now $h \in N_{j_{j(*)}+1} \subseteq N_{j}$ hence $h(\zeta) \in N_{j}$ hence $h(\zeta) \in N_{j} \cap \sigma \backslash f_{\delta_{j}}(\zeta)$, hence (by the definition of $g_{j}$ ), $g_{j}(\zeta) \leq h(\zeta)$ hence (as $j \in S$ ) we have $g^{*}(\zeta) \leq h(\zeta)$ but $h \in G \subseteq \prod_{\xi \in A} g^{*}(\xi)$, so $h(\zeta)<g^{*}(\zeta)$, contradiction. 


\section{$\S 4$ Entangled Orders - Narrow Order Boolean Algebra Revisited}

4.1 Theorem. 1) If $\kappa^{+4} \leq \operatorname{cf}(\lambda)<\lambda \leq 2^{\kappa}$ then there is an entangled linear order of cardinality $\lambda$.

2) Moreover, if $\chi_{0}<\lambda$ we can demand that the linear order has density character $\geq \chi_{0}$ (in fact, in every interval of the linear order).

Remark. See more [Sh 462] and [Sh 666].

Proof. Without loss of generality $\chi_{0}>\operatorname{cf}(\lambda) \geq \kappa^{+4}$. By [Sh 355, 2.1] there is an increasing continuous sequence $\left\langle\lambda_{i}: i<\operatorname{cf}(\lambda)\right\rangle$ of singular cardinals with limit $\lambda$ such that $\operatorname{tcf}\left(\prod_{i<\operatorname{cf}(\lambda)} \lambda_{i}^{+},<_{\left.J_{\operatorname{cf}(\lambda)}^{\mathrm{bd}}\right)}\right)=\lambda^{+}$and $\lambda_{0}>\chi_{0}$. The proof will be split to cases (one of them relies on the solution to others for smaller cardinals, so you may want to say we are proving 4.1 by induction on $\lambda$ ). Without loss of generality $\chi_{0}>\operatorname{cf}(\lambda)$.

Case I: For $i<\operatorname{cf}(\lambda)$ we have $\max \operatorname{pcf}\left\{\lambda_{j}^{+}: j<i\right\}<\lambda$.

So for some unbounded $A \subseteq \operatorname{cf}(\lambda)$ we have $i \in A \Rightarrow \lambda_{i}^{+}>\max \operatorname{pcf}\left\{\lambda_{j}^{+}: j \in\right.$ $A \cap i\}$.

So $\mathfrak{a}=\left\{\lambda_{i}^{+}: i \in A\right\}$ is as required in $\left[\right.$ Sh 355, 4.12] (with $\lambda^{+}, \operatorname{cf}(\lambda)$ here standing for $\lambda, \kappa$ there, noting that $2^{\operatorname{cf}(\lambda)} \geq 2^{\kappa} \geq \lambda$ ).

So we can assume:

\section{Assumption - not Case I}

So there is $\mu, \chi_{0}<\mu<\lambda, \operatorname{cf}(\mu)<\operatorname{cf}(\lambda), \operatorname{pp}_{<\operatorname{cf}(\lambda)}(\mu)>\lambda$. Choose a minimal such $\mu$, so by $3.2(2)$ :

$(*) \mathfrak{a} \subseteq \operatorname{Reg} \backslash \chi_{0} \& \sup (\mathfrak{a})<\mu \quad \& \quad|\mathfrak{a}|<\operatorname{cf}(\lambda) \Rightarrow \max \operatorname{pcf}(\mathfrak{a})<\lambda$

Clearly (by $[$ Sh $355,2.3]$ ) in $(*)$ 's conclusion we can replace " $<\lambda$ " by " $<\mu$ " i.e.

$(*)^{\prime} \mathfrak{a} \subseteq \operatorname{Reg} \backslash \chi_{0} \& \sup (\mathfrak{a})<\mu \quad \& \quad|\mathfrak{a}|<\operatorname{cf}(\lambda) \Rightarrow \max \operatorname{pcf}(\mathfrak{a})<\mu$

Let $\sigma=: \operatorname{cf}(\mu)$, so $\operatorname{pp}(\mu)=\operatorname{pp}_{<\operatorname{cf}(\lambda)}(\mu)$ (by $[$ Sh 371, 1.6(3)]) and remember $\operatorname{pp}_{<\operatorname{cf}(\lambda)}(\mu)>\lambda$.

Case II: $\sigma \geq \kappa$ (and not Case I; actually $2^{\sigma} \geq \mu$ suffices).

First assume $\sigma>\aleph_{0}$. As said above $\operatorname{pp}_{<\operatorname{cf}(\lambda)}(\mu)>\lambda$ and by [Sh 371, 1.7] there is a strictly increasing sequence $\left\langle\mu_{i}^{*}: i<\sigma\right\rangle$ of regular cardinals satisfying $\mu=\bigcup_{i<\sigma} \mu_{i}^{*}$, and $\lambda^{+}=\max \operatorname{pcf}\left\{\mu_{i}^{*}: i<\sigma\right\}=\operatorname{tcf} \prod_{i<\sigma} \mu_{i}^{*} / J_{\delta}^{\mathrm{bd}}$. Now as we can replace $\left\langle\mu_{i}^{*}: i<\sigma\right\rangle$ by $\left\langle\mu_{i}^{*}: i \in A\right\rangle$ for any $A \subseteq \sigma$ unbounded, by (*)' without loss of generality $\mu_{i}^{*}>\max \operatorname{pcf}\left\{\mu_{j}^{*}: j<i\right\}$, so we can apply again [Sh 355, 4.12] (or $3.2(5))$.

When $\sigma=\aleph_{0}, 4.1$ follows from [Sh 355, 4.13(1)]. 
So $\sigma^{+4} \leq \operatorname{cf}(\lambda)$. Let $\mathscr{P}=:\left\{A: A \subseteq \operatorname{cf}(\lambda)\right.$, otp $(A)=\kappa^{+}, A$ is a closed subset of $\sup (A)$ and $\left.\max \operatorname{pcf}\left\{\lambda_{i}^{+}: i \in c\right\}=\lambda_{\sup (A)}^{+}\right\}$. For any $C \in \mathscr{P}$, try to choose by induction on $i<\kappa^{+}\left(\mathfrak{b}_{i}\right)=\mathfrak{b}_{i}[C]$ and $\gamma_{i}=\gamma_{i}[C]$ such that:

(i) $\mathfrak{b}_{i} \subseteq \operatorname{Reg} \cap \mu \backslash \bigcup_{j<i} \mathfrak{b}_{j} \backslash \chi_{0}$

(ii) $\gamma_{i} \in C \backslash \bigcup_{j<i}\left(\gamma_{j}+1\right)$

(iii) $\lambda_{\gamma_{i}}^{+} \in \operatorname{pcf}\left(b_{i}\right)$

(iv) $\left|\mathfrak{b}_{i}\right| \leq \kappa$

(v) all members of $b_{i}$ are $\left(\chi_{0}, \kappa^{+}, \aleph_{0}\right)$-inaccessible

(vi) $\gamma_{i}$ is minimal under those requirements.

Subcase IIIa: For some $j<\kappa^{+}$.

For every $C \in \mathscr{P}$ such that $\operatorname{Min}(C) \geq j$. For some $\varepsilon(*)<\operatorname{cf}(\lambda)$, we cannot define $\mathfrak{b}_{i}, \gamma_{i}$ are defined iff $\varepsilon(*)$.

Let $C, i(*)$ be as above. Let $\gamma^{*}=\bigcup_{i<i(*)} \gamma_{i}$, so $\gamma^{*} \in C$. Now if $\gamma \in C \backslash \gamma^{*}$ then (by $\left[\right.$ Sh 355, 1.5B]) as $\operatorname{pp}_{\sigma}(\mu) \geq \lambda^{+}>\lambda_{\gamma}^{+}$, there is $\mathfrak{a}_{\gamma} \subseteq \operatorname{Reg} \cap\left(\chi_{0}, \mu\right),\left|\mathfrak{a}_{\gamma}\right| \leq \sigma$ such that $\lambda_{\gamma}^{+} \in \operatorname{pcf}\left(\mathfrak{a}_{\gamma}\right)$. By 3.2(3) there is $\mathfrak{c}_{\gamma} \subseteq \operatorname{Reg} \cap\left(\chi_{0}, \mu\right)$ of cardinality $\leq \kappa$ consisting of $\left(\chi_{0}, \kappa^{+}, \aleph_{0}\right)$-inaccessible cardinals such that $\lambda_{\gamma}^{+} \in \operatorname{pcf}\left(\mathfrak{c}_{\gamma}\right)$. Now $\gamma, c_{\gamma} \backslash \bigcup_{i<i(*)} \mathfrak{b}_{i}$ cannot serve as $\gamma_{i(*)}, \mathfrak{b}_{i(*)}$ so necessarily $\lambda_{\gamma}^{+} \notin \operatorname{pcf}\left(\mathfrak{c}_{\gamma} \backslash \bigcup_{i<i(*)} \mathfrak{b}_{i}\right)$ hence without loss of generality $\mathfrak{c}_{\gamma} \subseteq \bigcup_{i<i(*)} \mathfrak{b}_{i}$

Version 2: So $\left\{\lambda_{\gamma}^{+}: \gamma \in C \backslash i(*)\right\} \subseteq \operatorname{pcf}\left\{\bigcup_{i<i(*)} \mathfrak{b}_{i}\right\}$ and $\left|\bigcup_{i<i(*)} \mathfrak{b}_{i}\right| \leq \kappa$. By the proof of [Sh 400,4.2] we get a contradiction.

Subcase IIIb: For every $j<\operatorname{cf}(\lambda)$ there are $C \in \mathscr{P}$ with $\operatorname{Min}(C)>j$ such that for $C$, the pair $\left(\mathfrak{b}_{i}, \gamma_{i}\right)$ defined for every $i<\kappa^{+}$.

We shall now show

$\otimes$ for every $i(*)<\operatorname{cf}(\lambda)$ there is $\lambda^{\prime} \in \lambda \cap \operatorname{pcf}\left\{\lambda_{j}^{+}: j<\operatorname{cf}(\lambda)\right\} \backslash \lambda_{i(*)}$ such that $\operatorname{Ens}\left(\lambda^{\prime}, \lambda^{\prime}\right)$ (exemplified by linear order which has density character $>\chi_{0}$ in every interval).

$\underline{\text { Why } \otimes \text { is sufficient: }}$ We can for $i<\operatorname{cf}(\lambda)$, choose $\mu_{i}^{*}, \lambda_{i}<\mu_{i}^{*}=\operatorname{cf}\left(\mu_{i}^{*}\right) \in \lambda \cap$ $\operatorname{pcf}\left\{\lambda_{j}^{+}: j<\operatorname{cf}(\lambda)\right\}$, as required in $\otimes$. As $\bigwedge \mu_{i}^{*}<\lambda$ without loss of generality $\left\langle\mu_{i}^{*}: i<\operatorname{cf}(\lambda)\right\rangle$ is (strictly) increasing. We try to choose by $i$ induction on $\epsilon<\operatorname{cf}(\lambda), i(\epsilon)<\operatorname{cf}(\lambda)$ strictly increasing such that $\mu_{i(\epsilon)}^{*}>\max \operatorname{pcf}\left\{\mu_{i(\zeta)}^{*}: \zeta<\epsilon\right\}$.

Let $i(\epsilon)$ be defined iff $\epsilon<\epsilon(*)$. So $\epsilon(*)$ is $\leq \operatorname{cf}(\lambda)$ and is a limit ordinal $\lambda$ and $\max \operatorname{pcf}\left\{\mu_{i(\epsilon)}^{*}: \epsilon<\epsilon(*)\right\} \geq \lambda$ hence $\geq \lambda^{+}, \operatorname{but} \operatorname{pcf}\left\{\mu_{i(\varepsilon)}^{*}: \varepsilon<\varepsilon(*)\right\} \subseteq$ 
$\mu_{i(\epsilon)}^{*}>\max \operatorname{pcf}\left\{\mu_{i(\zeta)}^{*}: \zeta<\epsilon\right\}, \mu_{i(\epsilon)}^{*}$ is strictly increasing, and $\operatorname{Ens}\left(\mu_{i(\epsilon)}^{*}, \mu_{i(\epsilon)}^{*}\right)$ for $\varepsilon<\varepsilon(*)$. So applying [Sh 355, 4.12] we finish.

Why $\otimes$ holds: Let $i(*)<\operatorname{cf}(\lambda)$ be given. Choose $C \subseteq(i(*), \operatorname{cf}(\lambda))$ from $\mathscr{P}$ such that $\left(\mathfrak{b}_{\varepsilon}[C], \gamma_{\varepsilon}[C]\right)$ is defined iff $\varepsilon<\varepsilon[C]$ and $\varepsilon[C]<\kappa^{+}$. By the definition of $\mathscr{P}$ we have $\max \operatorname{pcf}\left\{\lambda_{\gamma}^{+}: \gamma \in C\right\}<\lambda$. Let $\mathfrak{d}=:\left\{\lambda_{\gamma}^{+}: \gamma \in C\right\}$, let $\left\langle\mathfrak{b}_{\theta}[\mathfrak{d}]: \theta \in \operatorname{pcf}(\mathfrak{d})\right\rangle$ be as in [Sh 371, 2.6]. Let $\theta$ be minimal such that $\operatorname{otp}\left(\mathfrak{b}_{\theta}[\mathfrak{d}]\right)$ is $\geq \kappa$. We can find $B_{\epsilon} \subseteq C$ (for $\epsilon<\kappa$ ) such that $\left\{\lambda_{\gamma}^{+}: \gamma \in B_{\epsilon}\right\} \subseteq \mathfrak{b}_{\theta}[\mathfrak{d}]$, otp $\left|B_{\epsilon}\right|=\kappa$ and the $B_{\epsilon}$ 's are pairwise disjoint. Clearly $\max \operatorname{pcf}\left\{\lambda_{\gamma}^{+}: \gamma \in B_{\epsilon}\right\}=\theta$ as $\left\{\lambda_{\gamma}^{+}: \gamma \in B_{\epsilon}\right\}$ is $\subseteq \mathfrak{b}_{\theta}[\mathfrak{d}]$, but is not a subset of any finite union of $\mathfrak{b}_{\theta^{\prime}}[\mathfrak{c}], \theta^{\prime}<\theta$. Now letting $\mathfrak{a}^{*}=: \cup\left\{\mathfrak{b}_{\varepsilon}[C]: \varepsilon<\varepsilon[C]\right\}$, there is (by [Sh 371, 2.6]) a subset $\mathfrak{a}$ of $\mathfrak{a}^{*}$ such that $\theta=\max \operatorname{pcf}(\mathfrak{a})$ but $\theta \notin \operatorname{pcf}\left(\mathfrak{a}^{*} \backslash \mathfrak{a}\right)$. Now as $\theta \in \operatorname{pcf}\left\{\lambda_{\gamma}^{+}: \gamma \in B_{\epsilon}\right\}, \lambda_{\gamma}^{+} \in \operatorname{pcf}\left(\mathfrak{b}_{\gamma}\right)$ we have (by [Sh 345a, 1.12]) $\theta \in \operatorname{pcf}\left(\bigcup \mathfrak{b}_{\gamma}\right)$ hence by the previous sentence

$\theta \in \operatorname{pcf}\left(\mathfrak{a} \cap \bigcup_{\gamma \in B_{\epsilon}} \mathfrak{b}_{\gamma}\right)$. Let $\mathfrak{c}_{\epsilon}=: \mathfrak{a} \cap \bigcup_{\gamma \in B_{\epsilon}} \mathfrak{b}_{\gamma}, \lambda^{\prime}=\theta$, we can apply [Sh 355, 4.12] and get that there is an entangled linear order of cardinality $\lambda^{\prime}$ (which is more than required, see [Sh 345b]); and, of course, $\lambda_{i(*)}<\lambda^{\prime} \in \lambda \cap \operatorname{pcf}\left\{\lambda_{j}: j<\operatorname{cf}(\lambda)\right\}$. The assumptions of [Sh 355, 4.12] holds as the $\mathfrak{c}_{\epsilon}$ are pairwise disjoint (by (i) above), $\theta \in \operatorname{pcf}\left\{\lambda_{\gamma}^{+}: \gamma \in B_{\epsilon}\left\{\subseteq \operatorname{pcf}\left(\bigcup_{\gamma \in B_{\epsilon}} \mathfrak{b}_{\gamma}\right)=\operatorname{pcf}\left(\mathfrak{c}_{\epsilon}\right)\right.\right.$ and $\left[\theta_{1} \in \mathfrak{a} \Rightarrow \max \operatorname{pcf}\left(\mathfrak{a} \cap \theta_{1}\right)<\right.$ $\left.\theta_{1}\right]$ as $\theta_{1}$ is $\left(\chi_{0}, \kappa^{+}, \aleph_{0}\right)$-inaccessible and $\theta=\lambda^{\prime} \geq \sup \left\{\lambda_{\gamma}^{+}: \gamma \in C\right\}>\lambda_{i(*)}>\chi_{0}$. So $\otimes$ holds and we finish Subcase IIIb hence Case III.

Case IV: $\operatorname{cf}(\lambda)>\kappa^{+4}$ and $\sigma \leq \kappa$ (and not Case I).

For each $\delta<\operatorname{cf}(\lambda)$ of cofinality $\kappa^{+4}$ we can apply the previous cases (or the induction hypothesis on $\lambda$ ) and get an entangled linear order of power $\lambda_{\delta}^{+}$. So $\otimes$ holds and we finish as in Subcase IIIb.

4.2 Claim. Assume $\kappa^{+4} \leq \theta=c f(\theta),\left\langle\lambda_{i}: i<\theta\right\rangle$ is a strictly increasing sequence of regular cardinals, $\theta<\lambda_{i} \leq 2^{\kappa}$ and $\lambda_{\theta}=t c f\left(\prod_{i<\theta} \lambda_{i} / J_{\theta}^{\mathrm{bd}}\right)$.

1) If $\sup _{i<\theta} \lambda_{i} \leqq 2^{\kappa}$ then there is an entangled linear order of cardinality $\lambda_{\theta}$.

2) Ens $\left(\lambda_{\theta}, 2^{\kappa}\right)$.

Remark. Remember that if there is an entangled linear order in $\lambda$ then $\operatorname{Ens}(\lambda, \lambda)$ (so [Sh 345b, 7(5)]).

Proof. Same proof as 4.1.

4.3 Claim. Assume

(i) $\lambda$ is regular, uncountable

(ii) $\kappa<\lambda \Rightarrow 2^{\kappa}<2^{\lambda}$

(iii) for some regular $\chi \leqq 2^{\lambda}$ there is no linear order of cardinality $\lambda$ with $\geqq \chi$ 
Then $\left(2^{<\lambda}<2^{\lambda}\right.$ and) for some $\mu$

(a) for every regular $\chi$ in $\left(2^{<\lambda}, 2^{\lambda}\right]$ (or even $\left.\left(\mu, 2^{\lambda}\right]\right)$ there is an entangled linear order of cardinality $\chi$ and density $\mu$,

(b) $\mu \in\left(\lambda, 2^{<\lambda}\right], c f(\mu)=\lambda, p p_{\Gamma(\lambda)}(\mu)=2^{\lambda}, \mu$ is $\left(\lambda, \lambda^{+}, 2\right)$-inaccessible

(the linear order is $\left(T,<_{\ell x}\right), T \subseteq \mu>2$ has $\leqq \mu$ nodes and $\geqq \chi \lambda$-branches).

[Saharon: see also [Sh 430, §3].

Proof. Note: $2^{<\lambda}<2^{\lambda}$ [if $(\exists \theta<\lambda)\left(2^{\theta}=2^{<\lambda}\right)$ by (ii), otherwise $\operatorname{cf}\left(2^{<\lambda}\right)=\lambda$ and by classical cardinal arithmetic, $\operatorname{cf}\left(2^{\lambda}\right)>\lambda$, hence $2^{<\lambda}<2^{\lambda}$. By [Sh 355, Lemma 5.11] if the conclusion fails then for every regular $\chi$ in $\left(2^{<\lambda}, 2^{\lambda}\right]$ there is $\mu, \lambda=\operatorname{cf}(\mu)<\mu \leqq 2^{<\lambda}, \operatorname{pp}_{\Gamma(\mu)}(\mu) \geqq \chi$. Choose a minimal $\mu$ such that $\lambda<$ $\mu \leqq 2^{<\lambda}, \operatorname{cf}(\mu) \leqq \operatorname{cf}(\lambda)$ and $\operatorname{pp}^{+}(\mu)>\chi$ (note: $\mu$ does not depend on $\chi$, by [Sh $355,2.3])$. So necessarily $\mu$ is $\left(\lambda, \lambda^{+}, 2\right)$-inaccessible. Let $\chi \in\left(\mu, 2^{\lambda}\right]$ be regular. As $\left(2^{<\lambda}\right)^{<\lambda}=2^{<\lambda}$ necessarily $\operatorname{cf}(\mu)=\lambda$, so by [Sh 371, 1.6(3)] there is a strictly increasing sequence $\left\langle\mu_{i}: i<\operatorname{cf}(\mu)=\lambda\right\rangle$ of regular cardinals, $\lambda<\mu_{i}<\mu, \mu=\sum_{i} \mu_{i}$ and $\chi=\operatorname{tcf}\left(\Pi \mu_{i} / J_{\mathrm{cf} \lambda}^{\mathrm{bd}}\right)$. As $\mu$ is $\left(\lambda, \lambda^{+}, 2\right)$-inaccessible without loss of generality $\mu_{i}>\max \operatorname{pcf}\left\{\mu_{j}: j<i\right\}$. So by [Sh 355,4.12] we finish.

4.4 Conclusion. 1) For a class of cardinals $\mu$, there is an entangled linear order of cardinality $\mu^{+}$.

2) Assume $\lambda$ is strong limit singular. Then for some successor cardinal in $\left(\lambda, 2^{\lambda}\right]$ there is an entangled linear order.

Proof. 1) By part (2).

2) If $\aleph_{\lambda+4}<2^{\lambda}$ then apply theorem 4.1 (with $\lambda, \aleph_{\lambda+4}$ here standing for $\kappa, \lambda$ there) so there is an entangled linear order of cardinality $\aleph_{\lambda+4}+1\left(\leqq 2^{\lambda}\right)$, which is as required. So assume $2^{\lambda} \leqq \aleph_{\lambda+4}$. We know that there is a linear order of cardinality $2^{\lambda}$ and density character $\lambda$; hence (see [Sh:g, AP,§1]) there is an entangled linear order of cardinality $\operatorname{cf}\left(2^{\lambda}\right)$. But as $2^{\lambda} \leqq \aleph_{\lambda^{+4}}$ necessarily $\operatorname{cf}\left(2^{\lambda}\right)$ is a successor cardinal. 


\section{$\S 5$ PRD: Measuring $\Pi f(i)$ By a Family $\Gamma$ OF IDEALS AND FAMILY SEQUENCES $\left\langle B_{i}: i<\kappa\right\rangle,\left|B_{i}\right|<\mu_{i}$}

In [Sh 371, $\S 4$, and here in $\S 1$ we have dealt with generalizations of the measuring $\prod_{i<\kappa} f(i) / I$, i.e. whereas defining $\operatorname{cov}(\lambda, \lambda, \theta, \sigma)$ we cover a set $a \in[\lambda]^{<\theta}$ by $<\sigma$ subsets of cardinality $<\lambda$; there we ask that $\kappa$ belongs to the closure to a normal ideal of $J$ union the family of $A \subseteq \kappa$ for which we succeed to cover. Here we replace "normal" by an abstract property $\Gamma$ (and phrase the required properties). We also generalize normality to ideals on $\mathscr{Y}$ with $\iota: \mathscr{Y} \rightarrow \kappa$, a generalization used in [Sh $420, \S 4]$, [Sh 430].

5.1 Context. 1) $\kappa$ is a regular uncountable cardinal, $\mathscr{Y}$ a set, $\iota$ a function from $\mathscr{Y}$ onto $\kappa, \mathscr{Y}_{i}=\iota^{-1}(\{i\})$. Here $I, J$ vary on ideals on $\mathscr{Y}, \Gamma$ a family of proper ideals on $\mathscr{Y}$.

5.2 Definition. 1) $\Gamma_{\mathscr{Y}, \kappa, \sigma}=\{J: J$ a $\sigma$-complete ideal on $\mathscr{Y}\}$ (if $(\mathscr{Y}, \iota)=\left(\kappa, \operatorname{id}_{\kappa}\right)$ this $\Gamma_{\kappa, \sigma}$ is essentially $\left.\Gamma\left(\kappa^{+}, \sigma\right)\right)$.

2) $\Gamma_{\mathscr{Y}, \kappa}=\Gamma_{\mathscr{Y}, \kappa}^{\text {nor }}=:\{J: J$ a normal ideal on $\mathscr{Y}\}$ (normal - see 5.3(0) below). If $\mathscr{Y}=\kappa, \iota=$ id we write $\Gamma_{\kappa}^{\text {nor }}$.

5.3 Definition. 0) An ideal $I$ on $\mathscr{Y}$ is normal if: for any club $C$ of $\kappa$ the set $\bigcup_{i \notin C} \mathscr{Y} i$ belong to $I$ and for any sequence $\left\langle A_{i}: i<\kappa\right\rangle$ of sets from $I$ the set $\nabla_{i} A_{i}=:\{x \in$ $\left.\mathscr{Y}: x \in \bigcup_{j<\iota(x)} A_{j}\right\}$ belongs to $I$. (So normal implies $\kappa$-complete).

1) We say $\Gamma$ is $\sigma$-complete if every $J \in \Gamma$ is $\sigma$-complete.

2) We say $\Gamma$ is normal if every $J \in \Gamma$ is normal.

3) We say $\Gamma$ is restriction closed when: $J \in \Gamma, A \subseteq \kappa, A \neq \emptyset \bmod J$ implies there is $I \in \Gamma, J \cup\{\kappa \backslash A\} \subseteq I$.

4) We say $\Gamma$ is closed if for every $\mathscr{P} \subseteq \mathscr{P}(\mathscr{Y}), c \ell_{\Gamma}(\mathscr{P})$ is well defined where $c \ell_{\Gamma}(\mathscr{P})$ is the minimal member of $\Gamma \cup\{\mathscr{P}(\kappa)\}$ which include it, i.e. $(\forall I \in \Gamma)[\mathscr{P} \subseteq I \Leftrightarrow$ $\left.c \ell_{\Gamma}(\mathscr{P}) \subseteq I\right]$.

Note: $c \ell_{\Gamma}$ for $a$ not necessarily closed $\Gamma$, is a partial function.

5) We say $\Gamma$ has character $\leqq \mu$ when: $\mathscr{P}(\kappa)=c \ell_{\Gamma}(\mathscr{P})$ where $\mathscr{P} \subseteq \mathscr{P}(\kappa)$ implies that for some $\mathscr{P}^{\prime} \subseteq \mathscr{P}$ of cardinality $\leqq \mu$, we have $\mathscr{P}(\kappa)=c \ell_{\Gamma}(\mathscr{P})$.

6) The character of $\Gamma$ is the minimal cardinal $\mu$ such that $\Gamma$ has character $\leqq \mu$.

5.4 Definition. 1) We say $\Gamma$ is suitable if it is $\theta$-suitable for every $\theta$ ? We say that $\Gamma$ is $\theta$-suitable when: for every ideal $J \in \Gamma$ on $\mathscr{Y}$, if then $\mathscr{P}(\kappa)=c \ell_{\Gamma}\left(J \cup\left\{f_{\eta}^{-1}(\{0\})\right.\right.$ : $\eta \in T\})$ where

( $i) T$ is a (non-empty) set of finite sequences of ordinals $<\theta$ closed under initial segments

(ii) $A_{\eta} \subseteq \mathscr{Y}$ for $\eta \in T$ and $A_{<>}=\mathscr{Y}$

(iii) for each $\eta \in T$ of length $n$, 
(iv) $\eta \triangleleft \nu \Rightarrow A_{\eta} \supseteq A_{\nu}$

(v) $f_{\eta}: A_{\eta} \rightarrow$ Ord

(vi) if $\eta^{\wedge}<\zeta>\in T$ and $y \in A_{\eta^{\wedge}<\zeta>}, f_{\eta}(y) \neq 0$ then $f_{\eta}(y)>f_{\eta^{\wedge}<\zeta>}(y)$.

5.5 Remark. 1) Clearly for $\theta_{1} \leqq \theta_{2}$ if $\Gamma$ is $\theta_{2}$-suitable then $\Gamma$ is $\theta_{1}$-suitable.

2) If $\theta \geqq 2^{|\mathscr{Y}|}$ its value is immaterial, so we can omit it.

5.6 Claim. 1) $\Gamma_{\mathscr{Y}, \kappa, \sigma}$, if $\kappa \geqq \sigma=\operatorname{cf}(\sigma)>\aleph_{0}$ is closed, restriction closed, of character $\sigma$ and is suitable. For $\mathscr{P} \subseteq \mathscr{P}(\mathscr{Y})$,

$$
c \ell_{\Gamma_{\mathscr{Y}, \kappa, \sigma}}(\mathscr{P})=\left\{Z: \text { for some } \alpha<\sigma, A_{i} \in \mathscr{P} \text { for } i<\alpha \text { we have } Z \subseteq \bigcup_{i<\alpha} A_{i}\right\}
$$

2) If $\mathscr{P}$ is a family of subsets of $\mathscr{Y}, \Gamma=\Gamma_{\mathscr{Y}, \kappa}^{\mathrm{nor}} \underline{\text { then }}$

$$
\begin{gathered}
c \ell_{\Gamma}(\mathscr{P})=\{Z: Z \subseteq \mathscr{Y} \text { and for some club } C \text { of } \kappa \text { and sequence } \\
\left\langle A_{i}: i<\kappa\right\rangle \text { of member of } \mathscr{P} \text { we have: } \\
Z \subseteq\left\{x \in \mathscr{Y}: \iota(x) \in C \text { and } x \in \bigcup_{j<\iota(x)} A_{j}\right\} .
\end{gathered}
$$

3) $\Gamma_{\mathscr{Y},{ }_{\kappa}}^{\text {nor }}$ (remember $\left.\kappa=c f(\kappa)>\aleph_{0}\right)$ is closed, restriction closed, suitable and of character $\kappa$.

4) If $\Gamma$ is $\theta$-suitable and has character $\leqq \theta$ then it is suitable (we shall use this freely).

Proof. 1) Let us check suitability leaving the rest to the reader; so let $J, \theta,\left\langle A_{\eta}, f_{\eta}\right.$ : $\eta \in T\rangle$ be as in Definition 5.4. If the required conclusion in Definition 5.4 fails then there is a $\sigma$-complete filter $I$ on $\kappa$ containing $J \cup\left\{f_{\eta}^{-1}(\{0\}): \eta \in T\right\}$. For each $\eta$, by condition (iii), for some set $w_{\eta}$ of ordinals, $\left|w_{\eta}\right|<\sigma$ and $B_{\eta} \in J$ we have

$(*) \mathscr{Y}=B_{\eta} \cup\left\{\kappa \backslash A_{\eta \uparrow 0} \backslash \ldots \backslash A_{\eta \uparrow \ell g \eta}\right\} \cup\left\{A_{\eta^{\wedge}<\zeta>}: \zeta \in w_{\eta}\right\}$ (and $\zeta \in w_{\eta} \Rightarrow \eta^{\wedge}<\zeta>\in T$ ).

Let $T^{*}=:\left\{\eta \in T: \ell<\ell g(\eta) \Rightarrow \eta(\ell) \in w_{\eta \mid \ell}\right\}$, as $\sigma$ is regular uncountable, clearly $\left|T^{*}\right|<\sigma$. Let

$$
B=: \cup\left\{B_{\eta}: \eta \in T^{*}\right\} \cup\left\{A_{\eta}: \eta \in T^{*} \text { and } A_{\eta} \in I\right\} \cup\left\{f_{\eta}^{-1}(\{0\}): \eta \in T^{*}\right\} .
$$

Now $B$ is the union of $<\sigma$ members of $I$ and $I$ is $\sigma$-complete, so $B \in I$. Choose $y \in \kappa \backslash B$. 


$$
\begin{gathered}
\eta_{0}=<> \\
\eta_{n} \in T^{*}, \\
\lg \left(\eta_{n}\right)=n \\
\eta_{n} \unlhd \eta_{n+1} \\
y \in A_{\eta_{n}} .
\end{gathered}
$$

For $n=0, \eta_{0}=<>$ so $y \in \mathscr{Y}=A_{<>}$. For $n+1, y \in A_{\eta_{n}\lceil n}, \eta_{n} \in T^{*}$ and $y \notin B$ hence $y \notin B_{\eta_{n}}$, so by $(*)$ there is $\zeta_{n} \in w_{\eta_{n}}$ such that $y \in A_{\eta_{n}{ }^{\wedge}<\zeta_{n}>}$ so $\eta_{n+1}=\eta_{n} \wedge\langle\zeta\rangle$ is as required.

In the end for each $n$ we have $f_{\eta_{n}}(y)>0$ as $f_{\eta_{n}}^{-1}(\{0\}) \subseteq B$, (remember $\left.\eta_{n} \in T^{*}\right)$ hence by condition (vi) from 5.4, $\left\langle f_{\eta_{n}}(y): n<\omega\right\rangle$ is a strictly decreasing sequence of ordinals, contradiction.

2) Left to the reader.

3) Again we leave the proof of restriction closed and closed and having character $\kappa$ to the reader and prove suitability. The proof is similar but use diagonal union. So by part (2) and condition (iii) of 5.4 for each $\eta \in T$ for some $B_{\eta} \in J$ and function $h_{\eta}$ from $\kappa$ to ordinals such that $\eta^{\wedge}\left\langle h_{\eta}(i)\right\rangle \in T$ for $i<\kappa$, we have

$$
(*) \mathscr{Y}=B_{\eta} \cup\left\{\kappa \backslash A_{\eta \uparrow 0} \backslash A_{\eta \uparrow 1} \backslash \ldots \backslash A_{\eta\lceil\ell g \eta}\right\} \cup\left\{x \in \mathscr{Y}: x \in \bigcup_{i<\iota(x)} A_{\eta^{\wedge}\left\langle h_{\eta}(i)\right\rangle}\right\}
$$

By renaming, without loss of generality $T \subseteq{ }^{\omega>} \kappa$ and each $h_{\eta}$ the identity function.

Let $I$ be the normal ideal on $\mathscr{Y}$ generated by $J \cup\left\{f_{n}^{-1}(\{0\}): \eta \in T\right\}$, so we assume $I$ is a normal proper ideal and we shall get a contradiction.

Now define $\mathscr{Y}^{*}$

$$
\begin{aligned}
\mathscr{Y}^{*}=\{x \in \mathscr{Y}: & (a) \quad \iota(x) \text { a limit ordinal }<\kappa \\
& (b) \quad \text { if } \eta \in T \cap^{\omega>} \iota(x) \text { then } x \notin B_{\eta} \\
& \left.(c) \quad \text { if } \eta \in T \cap{ }^{\omega>} \iota(x) \text { and } A_{\eta} \in I \text { then } x \notin A_{\eta}\right\} .
\end{aligned}
$$

Clearly $\mathscr{Y}^{*} \equiv \mathscr{Y} \bmod I$, hence we can find $x(*) \in \mathscr{Y}^{*}$. Now we choose by induction on $n \eta_{n} \in{ }^{\omega>} \iota(x(*))$ as in the proof of part (1) and get similar contradiction.

4) Left to the reader. 
5.7 Definition. 1) For $\bar{\mu}=\left\langle\mu_{i}: i<\kappa\right\rangle, J$ an ideal on $\mathscr{Y}$ and $f: \mathscr{Y} \rightarrow$ ord we define

$\operatorname{prd}_{J}^{\Gamma}(f, \bar{\mu})=\operatorname{Min}\left\{|\mathscr{P}|: \mathscr{P}\right.$ is a family of sequences of the form $\left\langle B_{x}: x \in \mathscr{Y}\right\rangle$, each $B_{x}$ a set of ordinals, $B_{x}$ of cardinality $<\mu_{\iota(x)}$ such that for every $g \in{ }^{x}$ ord satisfying $g \leqq f$, we have :

$$
\left.\mathscr{P}(\kappa)=c \ell_{\Gamma}\left[J \cup\left\{\left\{x \in \mathscr{Y}: g(x) \in B_{x}\right\}:\left\langle B_{x}: x \in \mathscr{Y}\right\rangle \in \mathscr{P}\right\}\right]\right\} .
$$

2) If above $J \subseteq I, I$ an ideal on $\kappa$

$\operatorname{prd}_{J, I}^{\Gamma}(f, \bar{\mu})=\operatorname{Min}\left\{|\mathscr{P}|: \mathscr{P}\right.$ is a family of sequences $\left\langle B_{x}: x \in \mathscr{Y}\right\rangle$, each $B_{x}$ a set of ordinals of cardinality $<\mu_{\iota(x)}$ and for every $g \in{ }^{x}$ ord satisfying $q \leqq f$, we have :

$\left.I \subseteq c \ell_{\Gamma}\left(J \cup\left\{\left\{x \in \mathscr{Y}: g(x) \in B_{x}\right\}:\left\langle B_{x}: x \in X\right\rangle \in \mathscr{P}\right\}\right)\right\}$.

3) If $\bar{\mu}$ is constantly $\mu$, we may write $\mu$ instead. We can use also $\bar{\mu}=\left\langle\mu_{x}: x \in \mathscr{Y}\right\rangle$, (but usually do not) with the obvious meaning.

5.8 Claim. 1) If $\{x \in \mathscr{Y}: f(x)<\omega\} \in J$ we can in 5.7 demand $g<_{J} f$, and if in addition $\bigwedge_{x} f(x) \neq 0$ we can demand $g<f$ (without changing the values).

2) If $\left\{x: f(x) \geqq \mu_{\iota(x)}\right\} \in J \underline{\text { then }} \operatorname{prd}_{J}^{\Gamma}(f, \bar{\mu})=1$.

3) If $\bigwedge \mu_{i}=\mu, \operatorname{cf}\left(\mu_{>}|\mathscr{Y}|,\{x: f(x)>\mu\} \in J \underline{\text { then }} \operatorname{prd}_{J}^{\Gamma_{J}}(f, \bar{\mu}) \leqq \operatorname{cf}(\mu)\right.$.

4) If $f_{1} \leqq J f_{2}$ or just $\left\{x:\left|f_{1}(x)\right|>\left|f_{2}(x)\right|\right\} \in J \underline{\text { then }} \operatorname{prd}_{J}^{\Gamma_{J}}\left(f_{1}, \bar{\mu}\right) \leqq \operatorname{prd}_{J}^{\Gamma_{J}}\left(f_{2}, \bar{\mu}\right)$ (and the other obvious monotonicity properties).

5) If $\mu_{i}=\mu, \operatorname{cf}(\mu)>|\mathscr{Y}|$ we can in Definition 5.7 demand $\bigwedge_{x \in \mathscr{Y}} B_{x}=B_{0}$ for $\bar{B} \in \mathscr{P}$ (i.e. without changing the value).

6) If $\Gamma$ is $\sigma$-complete and restriction closed, $\epsilon(*)<\sigma,\left\langle A_{\epsilon}: \epsilon<\epsilon(*)\right\rangle$ is a partition of $\mathscr{Y}(J, \bar{\mu}$ as in 5.7) then

$$
\sup _{\epsilon} \operatorname{prd}_{J+\left(\kappa \backslash A_{\epsilon}\right)}^{\Gamma}(f, \bar{\mu}) \leqq \operatorname{prd}_{J}^{\Gamma}(f, \bar{\mu}) \leqq \sum_{\epsilon} \operatorname{prd}_{J+\left(\kappa \backslash A_{\epsilon}\right)}^{\Gamma}(f, \bar{\mu}) .
$$

7) If $\Gamma$ is normal and restriction closed, $A_{\epsilon} \subseteq\{x \in \mathscr{Y}: \iota(x)>\epsilon\}$ for $\epsilon<\kappa,\left\langle A_{\epsilon}\right.$ : $\epsilon<\kappa\rangle$ a partition of $\mathscr{Y} \backslash \iota^{-1}(\{0\})$ then

$$
\sup _{\epsilon<\kappa} \operatorname{prd}_{J+\left(\mathscr{Y} \backslash A_{\epsilon}\right)}^{\Gamma} \leqq \operatorname{prd}_{J}^{\Gamma}(f, \bar{\mu}) \leqq \sum_{\epsilon<\kappa} \operatorname{prd}_{J+\left(\mathscr{Y} \backslash A_{\epsilon}\right)}^{\Gamma}(f, \bar{\mu}) .
$$

8) $\operatorname{prd}_{J}^{\Gamma_{\kappa}^{\text {nor }}}(f, \bar{\mu})=\operatorname{prc}_{J}(f, \bar{\mu})$ if $J \in \Gamma_{\kappa}^{\text {nor }}$. 
for each $i, \operatorname{cf}\left(\mu_{i}^{1}\right)<\mu_{i}^{1} \& \mu_{1}^{2}=\left(\mu_{i}^{1}\right)^{+}$. Then for (any $\Gamma, J \in \Gamma$ and $f \in{ }^{\mathscr{Y}}$ Ord) we have $\operatorname{prd}_{J}^{\Gamma}\left(f, \bar{\mu}^{1}\right)=\operatorname{prd}_{J}^{\Gamma}\left(f, \bar{\mu}^{2}\right)$.

10) If $\bar{\mu}=\left\langle\mu_{i}: i<\kappa\right\rangle$ is increasing continuous with limit $\mu$, and $\Gamma$ is normal, $J \in \Gamma$ then $\operatorname{prd}_{J}^{\Gamma}(\bar{\mu}, \bar{\mu}) \leqq \mu$ and even $\operatorname{prd}_{J}^{\Gamma}(\bar{\mu}, \bar{\mu})=\operatorname{cf}(\mu)$.

11) If $\bigwedge_{i<\kappa} \mu_{i}=\mu, \operatorname{cf}(\mu)>|\mathscr{Y}|, \Gamma=\Gamma_{\mathscr{Y}, \kappa, \sigma}$ then for any $\alpha, \operatorname{prd}_{\{\emptyset\}}^{\Gamma}(\alpha, \bar{\mu})=$ $\operatorname{cov}\left(|\alpha|, \mu,|\mathscr{Y}|^{+}, \sigma\right)$.

Proof. E.g.

6) The first inequality should be clear. Also the second: assume it fails, let $\lambda_{\epsilon}=$ $\operatorname{prd}_{J+\left(\kappa \backslash A_{\epsilon}\right)}^{\Gamma_{1}}(f, \bar{\mu}), \sum_{\epsilon<\epsilon(*)} \lambda_{\epsilon}<\operatorname{prd}_{J}^{\Gamma}(f, \bar{\mu})$, let $\mathscr{P}_{\epsilon}$ exemplify the definition of $\lambda_{\epsilon}$ and $\mathscr{P}$ be $\bigcup_{\epsilon<\epsilon(*)} \mathscr{P}_{\epsilon}$. As $\sum_{\epsilon} \lambda_{\epsilon}<\operatorname{prd}_{J}^{\Gamma}(f, \bar{\mu}), \mathscr{P}$ cannot exemplify $\sum_{\epsilon} \lambda_{\epsilon} \geqq \operatorname{prd}_{J}^{\Gamma}(f, \bar{\mu})$, so there is a function $g \in{ }^{\kappa}$ ord exemplifying this, so there is a proper ideal $I \in \Gamma$ extending

$$
J \cup\left\{\left\{x \in \mathscr{Y}: g(x) \in B_{x}\right\}:\left\langle B_{x}: x \in \mathscr{Y}\right\rangle \in \mathscr{P}\right\}
$$

As $\Gamma$ is $\sigma$-complete also $I$ is $\sigma$-complete so for some $\epsilon<\epsilon(*)$ we have $A_{\epsilon} \notin I$; but $\Gamma$ is restriction closed so there is $I_{1} \in \Gamma, I \cup\left\{\mathscr{Y} \backslash A_{\epsilon}\right\} \in I_{1}$. So $I_{1} \in \Gamma$ extend

$$
\left[J \cup\left\{\mathscr{Y} \backslash A_{\epsilon}\right\}\right] \cup\left\{\left\{x \in \mathscr{Y}: g(x) \in B_{x}\right\}:\left\langle B_{x}: x \in \mathscr{Y}\right\rangle \in \mathscr{P}_{\epsilon}\right\}
$$

contradicting the choice of $\mathscr{P}_{\epsilon}$.

5.9 Lemma. 1) Suppose

(*) $\mu_{i}=\mu=\operatorname{cf}(\mu)>|\mathscr{Y}|, \Gamma$ is suitable, restriction closed, $f \in{ }^{\mathscr{Y}}$ Ord and $J$ an ideal on $\kappa$.

Then:

$\otimes$

$$
\begin{array}{r}
\mu+\operatorname{prd}_{J}^{\Gamma}(f, \bar{\mu})=\mu+\sup \left\{\operatorname{tcf} \prod_{x \in \mathscr{Y}} \lambda_{x} / I: I \text { an ideal on } \mathscr{Y} \text { in } \Gamma\right. \\
\text { extending } J \text { such that } \\
\left.\mu<\lambda_{x}=c f\left(\lambda_{x}\right) \leqq f(x)\right\} .
\end{array}
$$

2) If $\Gamma$ is normal, $\bar{\mu}=\left\langle\mu_{i}: i<\kappa\right\rangle, \mu_{i}=\theta_{i}^{+},\left\langle\theta_{i}: i<\kappa\right\rangle$ is increasing continuous, $\theta_{i}>\kappa, \mu_{i}>\left|\mathscr{Y}_{i}\right|, \mu=\bigcup_{i<\kappa} \mu_{i}, \Gamma$ suitable and restriction closed and $f \in{ }^{\mathscr{Y}}$ Ord, $J$ an ideal on $\mathscr{Y}$ then $(\otimes)$ above holds. 
5.10 Conclusion. 1) Suppose $\mu, \bar{\mu}, \Gamma, J$ are as in $5.9, f \in{ }^{\mathscr{Y}}$ ord and $\{x: \operatorname{cf}[f(x)]=$ $\left.f(x) \geqq \mu_{\iota(x)}\right\} \in J \underline{\text { then }}$

$$
\begin{aligned}
\mu+\operatorname{prd}_{J}^{\Gamma}(f, \bar{\mu})=\mu & +\sup \left\{\operatorname{prd}_{J}^{\Gamma}(g, \bar{\mu}): g<_{J} f\right\} \\
& +\sup \left\{\operatorname{tcf}_{x} \prod_{x \in \mathscr{Y}} f(x) / I: J \subseteq I \in \Gamma \text { (and the tcf well defined) }\right\}
\end{aligned}
$$

2) If in addition $G \subseteq\left\{g: g \in{ }^{\mathscr{Y}}\right.$ Ord, $\left.g<_{J} f\right\}$ is cofinal or at least $\mathscr{P}(\kappa)=$ $c \ell_{\Gamma}[I \cup\{\{i: h(i)<g(i)\}: g \in G\}]$ for every $h<_{J} f$, (and e.g. $\left.\bigwedge_{i} f(i) \geqq \theta\right) \underline{\text { then }}$

$$
\begin{aligned}
\mu+\operatorname{prd}_{J}^{\Gamma}(f, \bar{\mu})=\mu & +\sup \left\{\operatorname{prd}_{J}^{\Gamma}(g, \bar{\mu}): g \in G\right\} \\
& +\sup \left\{\operatorname{tcf} \prod_{x \in \mathscr{Y}} f(x) / I: J \subseteq I \in \Gamma\right\} .
\end{aligned}
$$

5.11 Claim. Suppose $\mu, \bar{\mu}, \Gamma, J$ are as in 5.9((1) or (2)), $g: \mathscr{Y} \rightarrow$ card and $f(x)=$ $g(x)^{+} \geqq \mu_{\iota(x)}^{+}$.

$$
\text { Then } \operatorname{prd}_{J}^{\Gamma}(f, \bar{\mu}) \leqq\left[\operatorname{prd}_{J}^{\Gamma}(g, \bar{\mu})\right]^{+}+\mu \text {. }
$$

Proof. Let $\mathscr{P}$ exemplify the value of $\operatorname{prd}_{J} \Gamma_{J}(g, \bar{\mu})$, say $|\mathscr{P}|=\chi$. So for every $h<_{J} f$, clearly $\{x \in \mathscr{Y}:|h(x)| \leqq g(x)\}=\mathscr{Y} \bmod J$, hence there is $\mathscr{P}_{h} \subseteq \prod_{x \in \mathscr{Y}} h(x)$ exemplifying $\operatorname{prd}_{J}^{\Gamma}(h, \bar{\mu}) \leqq \operatorname{prd}_{J}^{\Gamma}(g, \bar{\mu})=\chi$. Assume $\operatorname{prd}_{J}(f, \bar{\mu})>\chi^{+}+\mu$; by 5.9 there is $f^{\prime}: \mathscr{Y} \rightarrow$ ord, each $f^{\prime}(x)$ a regular cardinal $\geqq \mu_{i}, f^{\prime} \leqq f \bmod I$ where $I \in \Gamma$ an ideal extending $J$ such that $\chi^{+}<\operatorname{tcf} \prod_{x \in \mathscr{Y}} f^{\prime}(x) / I$. Let $\left\langle h_{\zeta}: \zeta<\chi^{\prime}\right\rangle$ be $<_{I}$-increasing cofinal in $\prod_{x \in \mathscr{Y}} f^{\prime}(x) / I$. As in $[$ Sh 355, 1.5] without loss of generality for some $\zeta(*)<\operatorname{tcf} \prod_{x \in \mathscr{Y}} f^{\prime}(x) / I$ of cofinality $\chi^{+}$we have: $\left\langle h_{\zeta}: \xi<\zeta(*)\right\rangle$ has a $<_{I^{-}}$lub $h^{\prime}$ such that: for 5.9(1) $\{x \in \mathscr{Y}: \operatorname{cf}[f(x)] \leqq \mu\} \in I$ and for $5.9(2)\{x \in \mathscr{Y}:$ $\left.\operatorname{cf}[f(x)]<\mu_{\iota(x)}\right\} \in I$; without loss of generality it is $h_{\zeta(*)}$ and $\bigwedge_{x \in \mathscr{Y}} \operatorname{cf}\left[h_{\zeta(*)}(x)\right] \geqq$ $\mu_{\iota(x)}$, and without loss of generality: $\xi<\zeta(*) \Rightarrow h_{\xi}<h_{\zeta(*)}$. For each $\bar{B}=\left\langle B_{x}\right.$ : $x \in \mathscr{Y}\rangle \in \mathscr{P}_{h_{\zeta(*)}}$ define a function $f_{\bar{B}}: f_{\bar{B}}(x)=\sup \left(h_{\zeta(*)}(x) \cap B_{x}\right)$. So $f_{\bar{B}}<h_{\zeta(*)}$ hence for some $\xi(\bar{B})<\zeta(*)$ we have $f_{\bar{B}}<f_{\xi(\bar{B})} \bmod I$. Let $\bigcup_{\bar{B}} \xi(\bar{B})<\xi<\zeta(*)-$ possible as the number of $\bar{B}$ 's is $\leqq\left|\mathscr{P}_{h_{\zeta(*)}}\right| \leqq \chi<\chi^{+}=\operatorname{cf}(\zeta(*))$. So for $\bar{B} \in \mathscr{P}_{h_{\zeta(*)}}$ we have $\left\{x \in \mathscr{Y}: f_{\xi}(x) \in B_{x}\right\} \in I$. But $f_{\xi}<f_{\zeta(*)}$ so we get contradiction to the choice of $\mathscr{P}_{h_{\zeta(*)}}$. 
5.12 Definition. Let $1-\operatorname{cf}_{J}^{\Gamma}\left(\left\langle a_{x}: x \in \mathscr{Y}\right\rangle\right)$ be

$$
\sup \left\{\operatorname{tcf} \prod_{x \in \mathscr{Y}} \lambda_{x} / I: J \subseteq I \in \Gamma \text { and } \lambda_{x} \in a_{x} \text { for } x \in \mathscr{Y}\right\}
$$

5.13 Claim. $\bar{\mu}=\left\langle\mu_{i}: i<\kappa\right\rangle$ is non-decreasing, $\Gamma$ is a suitable restriction close family of ideals on $\mathscr{Y}, J \in \Gamma, f \in \mathscr{Y}$ ord and $\bigwedge_{x \in \mathscr{Y}} f(x) \geqq \mu_{\iota(x)}=c f \mu_{\iota(x)}$.

1) If $\lambda \leqq \operatorname{prd}_{J}^{\Gamma}(f, \bar{\mu})$ is regular, then for some $\left\langle\mathfrak{a}_{x}: x \in \mathscr{Y}\right\rangle$ we have:

(i) $\mathfrak{a}_{x} \subseteq \operatorname{Reg} \cap f(x)^{+} \backslash \mu_{\iota(x)}$

(ii) $\left|\bigcup_{x \in \mathscr{Y}} \mathfrak{a}_{x}\right|<\mu$ if $(*)$ of 5.9(1) and $\left|\mathfrak{a}_{x}\right|^{+}<\mu_{\iota(x)}$ when 5.9(2)'s assumptions hold

(iii) $\lambda=1-c f_{J}^{\Gamma}\left(\left\langle a_{x}: x \in \mathscr{Y}\right\rangle\right)$.

2) If $\lambda$ is inaccessible, $\Gamma, \sigma$-complete and $\left[\chi<\mu \Rightarrow \operatorname{cov}\left(\chi, \chi_{0}, \kappa, \sigma\right)<\lambda\right] \underline{\text { then }}$ without loss of generality $\left|\bigcup_{x \in \mathscr{Y}} a_{x}\right|<\chi_{0}$.

Proof. 1) Like the proof of 1.1.

2) Straight.

5.14 Claim. Assume the hypothesis of 5.9.

If $g \in{ }^{\mathscr{Y}}$ Ord, and each $g(s)$ is an ordinal $\geqq \mu_{\iota(x)}$ and $f(i)=\aleph_{g(i)}$ and let $\lambda=\operatorname{prd}_{J}^{\Gamma}(g, \bar{\mu})+\bigcup_{\alpha<\mu}|\alpha|^{+3}+|\mathscr{Y}| \underline{\text { then }} \operatorname{prd}_{J}^{\Gamma}(f, \bar{\mu}) \leqq \aleph_{\lambda^{+}}$.

Proof. Assume not, so $\operatorname{prd}_{J}^{\Gamma}(f, \bar{\mu}) \geqq \aleph_{\lambda^{+}+1}$ hence by 5.9 there is $I \in \Gamma, J \subseteq I$ and $f^{*} \leqq f$ such that: each $f^{*}(x)$ is a regular cardinal $\geqq \mu_{\iota(x)}$ and $\aleph_{\lambda^{+}+1} \leqq$ $\operatorname{tcf}\left(\prod_{x \in \mathscr{Y}} f^{*}(x) / I\right)$. By $\left[\right.$ Sh 355, 1.5] for each $\alpha<\lambda^{+}$such that $\aleph_{\alpha} \geqq|\mathscr{Y}|$ (e.g. $\alpha \geqq \lambda)$ there is $f_{\alpha}<_{I} f^{*}, f_{\alpha} \leqq f^{*}$, each $f_{\alpha}(x)$ a regular cardinal $>\mu_{\iota(x)}$ and $\operatorname{tcf}\left(\prod_{x \in \mathscr{Y}} f^{*}(x) / I\right)=\aleph_{\alpha+1}$. Clearly $\alpha \neq \beta \Rightarrow f_{\alpha} \neq f_{I} f_{\beta}$. Now let $f_{\alpha}=\aleph_{g_{\alpha}}$, so $g_{\alpha}<_{I} g, g_{\alpha} \leqq g$. Let $\mathscr{P}$ exemplify $\operatorname{prd}_{J}^{\Gamma}(g, \bar{\mu}) \leqq \lambda$, so for each $\alpha<\lambda^{+}$for some $\bar{B}^{\alpha} \in \mathscr{P},\left\{y: f_{\alpha}(y) \in\left\{\aleph_{j}: j \in B_{y}^{\alpha}\right\}\right\} \notin I$ (and, by 5.8(9), and normality without loss of generality $\left.\sup \left\{\left|B_{y}^{\alpha}\right|: y \in \mathscr{Y}\right\}<\sum_{i<\kappa} \mu_{i}\right)$. By 3.3 we get contradiction. $\square_{5.14}$ 


\section{$\S 6$ The Existence of Strongly Almost Disjoint Families}

See $[$ Sh $355, \S 0]$ on the history of the subject.

6.1 Theorem. Assume $J$ is an ideal on $\kappa, \kappa$ not the union of $\aleph_{0}$ members of $J, \mu>\kappa^{<\sigma}$ where

$\otimes \sigma=\kappa^{+}$or at least $\forall A \in J^{+} \exists B \in J^{+}[B \subseteq A$ \& $|B|<\sigma]$

and $\sigma=c f(\sigma)>\aleph_{0}$.

Then

$$
T_{J}^{3}(\mu)=T_{J}^{2}(\mu) \leqq T_{J}^{1}(\mu) \leqq T_{J}^{4}(\mu)
$$

where

$$
\begin{aligned}
& T_{J}^{1}(\mu)=T_{J}(\mu)=\sup \{|\mathscr{F}|: \mathscr{F} \text { is a family of functions from } \kappa \\
& \text { to } \left.\mu \text { such that for } f \neq g \text { from } \mathscr{F} \text { we have } f \neq{ }_{J} g\right\} .
\end{aligned}
$$

$T_{J}^{2}(\mu)=\sup \left\{\lambda:\right.$ there are $n_{i}<\omega$ for $i<\kappa$ and regular

$$
\begin{aligned}
& \lambda_{i, \ell}>\kappa^{<\sigma} \text { for } i<\kappa, \ell<n_{i} \text { such that }: \lambda_{i, \ell} \leqq \mu \\
& \text { and } \lambda \leqq \max \operatorname{pcf}\left\{\lambda_{i, \ell}: i<\kappa, \ell<n_{i}\right\} ; \text { moreover if } A \in J^{+} \\
& \left.(=\mathscr{P}(\kappa) \backslash J) \underline{\text { then }} \lambda \leqq \max \operatorname{pcf}\left\{\lambda_{i, \ell}: i \in A, \ell<n_{i}\right\}\right\} .
\end{aligned}
$$

$T_{J}^{3}(\mu)$ is defined similarly but for $A \in J^{+}$we demand:

$$
\begin{gathered}
\lambda=\max \operatorname{pcf}\left\{\lambda_{i, \ell}: i \in A, \ell<n_{i}\right\} \\
T_{J}^{4}(\mu)=\operatorname{Min}\left\{\sup _{s} T_{J+\left(\kappa \backslash A_{n}\right)}^{2}(\lambda): A_{n} \subseteq A_{n+1} \subseteq \kappa=\bigcup_{n<\omega} A_{n}, A_{n} \notin J\right\} .
\end{gathered}
$$

6.2 Remark. 1) Note that usually the four terms in the conclusion of the theorem are equal.

( $\alpha$ ) If $J$ is $\aleph_{1}$-complete then $T_{J}^{2}(\mu)=T_{J}^{4}(\mu)$ hence all are equal

( $\beta$ ) all terms are equal if for $\left\langle A_{n}: n<\omega\right\rangle$ such that $A_{n} \subseteq \kappa, A_{n} \notin J, A_{n} \subseteq$ $A_{n+1}, \kappa=\bigcup_{n} A_{n}$ we have: for some $n$ and $B \subseteq A_{n}$ we have $(\kappa, J),(B, J \cap$ 
2) The supremum in the definition of $T_{J}^{1}(\mu)$ is always obtained.

[Why? If $\mathscr{F}_{1}, \mathscr{F}_{2}$ are as there, $\mathscr{F}_{1}$ maximal $\left|\mathscr{F}_{1}\right|<\left|\mathscr{F}_{2}\right|$ then for every $f \in F_{2}$ there is $g_{f} \in \mathscr{F}$, etc.].

Proof. $\underline{T}_{J}^{2}(\mu)=T_{J}^{3}(\mu)$.

Trivially $T_{J}^{3}(\mu) \leqq T_{J}^{2}(\mu)$; for the other direction let $\lambda$ appear in the sup defining $T_{J}^{2}(\mu)$, as exemplified by $\left\langle<\lambda_{i, \ell}: \ell<n_{i}>: i<\kappa\right\rangle$; as $\max \operatorname{pcf}\left\{\lambda_{i, \ell}: i \in A, \ell<n_{i}\right\}$ is always regular, without loss of generality $\lambda$ is regular.

By [Sh 355, §1]; more elaborately, for some $\mathfrak{a} \subseteq \mathfrak{a}^{*}=:\left\{\lambda_{i, \ell}: \ell<n_{i}, i<\kappa\right\}$ we have $[\mathfrak{a} \neq \emptyset \Rightarrow \lambda=\max \operatorname{pcf}(\mathfrak{a})]$ and $\lambda \notin \operatorname{pcf}\left[\mathfrak{a}^{*} \backslash \mathfrak{a}\right]$ and $\mathfrak{a} \neq \mathfrak{a}^{*} \Rightarrow \lambda<$ $\max \operatorname{pcf}\left(\mathfrak{a}^{*} \backslash \mathfrak{a}\right)$. Define $\lambda_{i, \ell}^{\prime}$ to be $\lambda_{i, \ell}$ if $\lambda_{i, \ell} \in a^{*}$. If $\mathfrak{a} \neq \mathfrak{a}^{*}$ let $u^{*}=\{(i, \ell)$ : $\left.\left.\lambda_{i, \ell} \in \mathfrak{a}^{*} \backslash \mathfrak{a}\right)\right\}, J=\left\{u \subseteq u^{*}: \max \operatorname{pcf}\left\{\lambda_{(i, \ell)}:(i, \ell) \in u\right\}<\lambda\right\}$. By [Sh 355, 1.5] we can find regular $\lambda_{i, \ell}^{\prime}<\lambda_{i, \ell}$ for $(i, \ell) \in u^{*}$ such that $\lambda=\operatorname{tcf} \prod_{(i, \ell) \in u^{*}} \lambda_{i, \ell}^{\prime} / J$. Now $\left\langle\lambda_{i, \ell}^{\prime}: \ell<n_{i}, i<\kappa\right\rangle$ exemplifies $\lambda \leqq T_{J}^{3}(\mu)$.

$\underline{T_{J}^{3}(\mu) \leq T_{J}^{1}(\mu)}$. Very easy; of course, instead $F \subseteq{ }^{\kappa} \mu$ we can have $F \subseteq{ }^{\kappa} Y$ as long as $|Y| \leqq \mu$. For $\lambda,\left\langle<\lambda_{i, \ell}: \ell<n_{i}>: i<\kappa\right\rangle$ as in the definition of $T_{J}^{3}(\mu)$, let $a=\left\{\lambda_{i, \ell}: i<\kappa, \ell<n_{i}\right\}$ and $\left\langle f_{\alpha}: \alpha<\lambda\right\rangle$ be a sequence of members of $\Pi a$ which is $<_{J_{<\lambda}[a]}$-increasing and cofinal. Now let $Y={ }^{\omega>}(\mu+1)$ and for each $\alpha<\lambda$ we define $g_{\alpha} \in{ }^{\kappa} Y$ as follows:

$$
g_{\alpha}(i)=:\left\langle\lambda_{i, \ell}: \ell<n_{i}\right\rangle^{\wedge}\left\langle f_{\alpha}\left(\lambda_{i, \ell}\right): \ell<n_{i}\right\rangle
$$

We leave the checking to the reader. We now turn to the main case.

$T_{J}^{1}(\mu) \leq T_{J}^{4}(\mu)$. Let $\lambda$ be the right side expression $-T_{J}^{4}(\mu)$ (so clearly $\left.\lambda \geqq \mu\right), \chi=$ : $\beth_{3}(\lambda)^{+}$and for $\zeta \leqq \omega+1$ let: $M_{\zeta}^{*} \prec\left(H(\chi), \in,<_{\chi}^{*}\right),\left\|M_{\zeta}^{*}\right\|=\lambda, \lambda+1 \subseteq M_{\zeta}^{*}, \zeta<$ $\xi \leqq \omega+1 \Rightarrow M_{\zeta}^{*} \in M_{\xi}^{*}$. Suppose $F \subseteq{ }^{\kappa} \mu$ exemplify $T_{J}^{1}(\mu)>\lambda$ and we shall get a contradiction, without loss of generality $F \in M_{0}^{*}$. Clearly for every $f \in{ }^{\kappa} \mu$ we have: $\{g \in F: \neg g \neq J f\}$ has cardinality $\leqq \kappa^{<\sigma}$ (remember $\otimes$ ), hence necessarily there is $f^{*} \in F$ such that for every $g \in{ }^{\kappa} \mu \cap M_{\omega+1}^{*}$ (e.g. $g \in F \cap M_{\omega+1}^{*}$ ) we have $f^{*} \neq J g$. Moreover, if $A \subseteq \kappa, A \notin J, B \subseteq \mu,|B| \leqq \kappa^{<\sigma}$ then $\{f \in F:\{\alpha \in A: f(\alpha) \in B\} \notin$ $J\}$ has cardinality $\leqq \kappa^{<\sigma}$ (again, remember $\otimes$ ), so if in addition $A, B \in M_{\zeta}^{*}$ then $\left[f \in F \quad \& \quad\{\alpha \in A: f(\alpha) \in B\} \notin J \Rightarrow f \in M_{\zeta}^{*}\right]$.

We define by induction on $k<\omega, N_{k}^{a}, N_{k}^{b}, a_{k}, f^{k}$ such that:

(a) $N_{k}^{a} \prec M_{0}^{*}, N_{k}^{b} \prec\left(H(\chi), \in,<_{\chi}^{*}\right)$

(b) $N_{0}^{b}$ is the Skolem Hull of $\left\{f^{*}\right\} \cup\left\{i: i \leqq \kappa^{<\sigma}\right\}$ in $\left(\mathscr{H}(\chi), \in,<_{\chi}^{*}\right)$

(c) $N_{0}^{a}$ is the Skolem Hull of $\left\{i: i \leqq \kappa^{<\sigma}\right\}$ (in $\left(\mathscr{H}(\chi), \in,<_{\chi}^{*}\right.$ ), equivalently in $\left.M_{0}^{*}\right)$

(d) $N_{n+1}^{b}$ is the Skolem Hull of $N_{n}^{b} \cup\left\{f^{n}(\theta): \theta \in a_{n}\right\}$

(e) $N_{n+1}^{a}$ is the Skolem Hull of $N_{n}^{a} \cup\left\{f^{n}(\theta): \theta \in a_{n}\right\}$

$(f) \mathfrak{a}_{n}=N_{n}^{a} \cap \lambda^{+} \cap \operatorname{Reg} \backslash\left(\kappa^{<\sigma}\right)^{+}$ 
(h) if $\mathfrak{b} \subseteq \mathfrak{a}_{n}, \max \operatorname{pcf}(\mathfrak{b}) \leqq \lambda$ and $|\mathfrak{b}|<\sigma \underline{\text { then }}$

$f^{n}\left\lceil\mathfrak{b} \in\left\{\operatorname{Max}\left\{f_{\lambda_{\ell}, \alpha_{\ell}}^{c_{\ell}}\lceil\mathfrak{b}: \ell<n\}: n<\omega, \alpha_{\ell}<\max \operatorname{pcf}(\mathfrak{b})\right.\right.\right.$ and

$\lambda_{\ell} \in \operatorname{pcf}\left(\mathfrak{a}_{n}\right)$ and $\left.\mathfrak{c}_{\ell} \in\left\{\mathfrak{b}_{\theta}\left[\mathfrak{a}_{n}\right]: \theta \in \operatorname{pcf}\left(\mathfrak{a}_{n}\right)\right\}\right\}$

where $\mathfrak{b} \mapsto\left\langle f_{\alpha}^{\mathfrak{b}}: \alpha<\max \operatorname{pcf}(\mathfrak{b})\right\rangle$ for $\mathfrak{b} \subseteq \mathfrak{a}_{n}$ is a definable function (in $(\mathscr{H}(\chi), \epsilon$ ,$\left.<_{\chi}^{*}\right),\left\langle f_{\alpha}^{b}: \alpha<\max \operatorname{pcf}(\mathfrak{b})\right\rangle$ as in [Sh 371, §1].

By 2.3 (i.e. [Sh 371, §1]) there is no problem to do it, $N_{n}^{a} \prec N_{n}^{b}, N_{n}^{a} \prec M_{0}^{*}, N_{n}^{a} \prec$ $N_{n+1}^{a}, N_{n}^{b} \prec N_{n+1}^{b}$ and (as in [Sh 400,3.3A,5.1A]) we get $\bigcup_{n} N_{n}^{a}=\bigcup_{n} N_{n}^{b}$, hence $\operatorname{Rang}\left(f^{*}\right) \subseteq \bigcup_{n<\omega} N_{n}^{a}$. Now for each $i<\kappa$ let $m(i)=\min \left\{m: f^{*}(i) \in N_{m}^{a}\right\}$, and we can find finite $\mathfrak{e}(i) \subseteq \bigcup_{\ell<m(i)} \mathfrak{a}_{\ell}, y(i) \subseteq \kappa^{<\sigma}+1$ such that $f^{*}(i) \in M_{m(i)}^{e(i), y(i)}$ where for any $\mathfrak{e} \subseteq \bigcup_{n} \mathfrak{a}_{k}$ and $y \subseteq \kappa^{<\sigma}+1$ (we define by induction of $\ell$ ):

$M_{0}^{e, y}$ is the Skolem Hull of $y$ in $\left(\mathscr{H}(\chi), \in,<_{\chi}^{*}\right)$,

$M_{\ell+1}^{e, y}=$ Skolem Hull of $M_{\ell}^{e} \cup\left\{f^{m}(\theta): m \leqq \ell\right.$ and $\left.\theta \in e \cap M_{\ell}^{e}\right\}$.

Clearly: $\left[\mathfrak{e} \subseteq \bigcup_{m} \mathfrak{a}_{m} \quad \& \quad \mathfrak{e} \in M_{\ell}^{*} \quad \& \quad \max \operatorname{pcf}(\mathfrak{e}) \leqq \lambda \quad \& \quad \ell<\omega \Rightarrow M_{\ell}^{e, y} \in M_{\ell+1}^{*}\right]$, and $\left[\mathfrak{e} \subseteq \mathfrak{d} \quad \& \quad y \subseteq z \Rightarrow M_{\ell}^{\mathfrak{e}, y} \subseteq M_{\ell}^{d, z}\right]$ and $M_{\ell}^{e, y} \subseteq N_{\ell}^{a}$.

Let $A_{n}=\{i<\kappa: m(i) \leqq n\}$. Clearly $A_{n} \subseteq A_{n+1}, \kappa=\bigcup_{n<\omega} A_{n}$, but $\kappa$ is not the union of $\aleph_{0}$ members of $J$, so for some $n(*)<\omega,\left[n \geqq n(*) \Rightarrow A_{n} \notin J\right]$. It suffices to prove:

$(*)$ if $m(*)<\omega, A \subseteq \kappa, \bigwedge_{i \in A} m(i) \leqq m(*), A \notin J$ then max $\operatorname{pcf}\left(\bigcup_{i \in A} \mathfrak{e}(i)>\lambda\right.$

[as this means $n(*) \leqq n<\omega \Rightarrow T_{J+\left(\kappa \backslash A_{n}\right)}^{2}(\mu)>\lambda$, hence $\left\langle A_{n(*)+\ell}: \ell<\omega\right\rangle$ and $\langle e(i): i<\kappa\rangle$ exemplified $T_{J}^{4}(\mu)>\lambda$ contradiction].

We can replace $A$ by any subset which is not in $J$.

By the assumption $\otimes$ without loss of generality $|A|<\sigma$, and suppose $A$ contra$\operatorname{dicts}(*)$. Let $\mathfrak{e}^{*}=\bigcup_{i \in A} \mathfrak{e}(i), y^{*}=\bigcup_{i \in A} y(i)$. As $\sigma$ is infinite, clearly $\left|\mathfrak{e}^{*}\right|<\sigma,\left|y^{*}\right|<$ $\sigma, \bigwedge\left\|M_{n}^{\mathfrak{e}^{*}, y^{*}}\right\|<\sigma\left(\right.$ remember $\left.\sigma>\aleph_{0}\right)$.

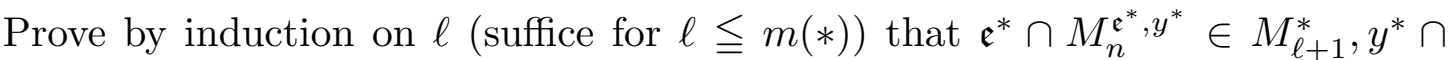
$M^{\mathfrak{e}^{*}, y^{*}} \in M_{\ell+1}^{*}$ and $M_{\ell}^{\mathfrak{e}^{*}, y^{*}} \subseteq M_{\ell+1}^{\mathfrak{e}^{*}, y^{*}}$. For $\ell=0$ this holds as $M_{0}^{\mathfrak{e}^{*}, y^{*}} \prec N_{0}^{a},\left\|M_{0}^{\mathfrak{e}^{*}, y^{*}}\right\|<$ $\sigma$ and $\mathfrak{e}^{*} \cap M_{0}^{\mathfrak{e}^{*}}=\mathfrak{e}^{*} \cap N_{0}^{a}$ is a subset of $N_{0}^{a}$ of cardinality $<\sigma, N_{0}^{a} \in M_{1}^{*},\left\|N_{0}^{a}\right\|^{<\sigma}=$ $\left\|N_{0}^{a}\right\| \leqq \kappa^{<\sigma}$. Similarly for $y^{*}$. For $\ell+1$, as we know $M_{\ell}^{\mathfrak{e}^{*}, y^{*}} \in M_{\ell+1}^{*}$ and $f^{\ell}\left\lceil\left(\mathfrak{e}^{*} \cap M_{\ell}^{\mathfrak{e}^{*}, y^{*}}\right) \in M_{\ell+1}^{*}\right.$ by (h) as max $\operatorname{pcf}\left(\mathfrak{e}^{*}\right) \leqq \lambda$ by an assumption hence $M_{\ell+1}^{\mathfrak{e}^{*}, y^{*}} \in M_{\ell+2}^{*}$. As $\left\|M_{\ell+1}^{\mathfrak{e}^{*}, y^{*}}\right\|=\kappa^{<\sigma},\left|\mathfrak{e}^{*}\right|<\sigma$ and $\kappa^{<\sigma}+1 \subseteq M_{0}^{*}$ necessarily $e^{*} \cap M_{\ell+1}^{e^{*}, y^{*}} \in M_{\ell+2}^{*}$. So $M_{m(*)}^{\mathrm{e}^{*}, y^{*}} \in M_{m(*)+1}^{*}$ so $\operatorname{Rang}\left(f^{*} \mid A\right) \subseteq M_{m(*)}^{\mathrm{e}^{*}, y^{*}} \in M_{m(*)+1}^{*}$, 
Proof of $6.1 A(1)$. $T_{J}^{4}(\mu) \leqq T_{J}^{1}(\mu)$. Let $A_{n} \subseteq \kappa, A_{n} \subseteq A_{n+1}, \kappa=\bigcup_{n<\omega} A_{n}, A_{n} \notin J$ and $T_{J+\left(\kappa \backslash A_{n}\right)}^{2}(\mu) \geqq \lambda$. For each $n$, by earlier parts of the proof, there is $\mathscr{F}_{n} \subseteq{ }^{\kappa} \mu$ such that $\left|\mathscr{F}_{n}\right| \geqq \lambda$ and $\left[f \neq g \in F_{n} \Rightarrow f \neq \neq_{J+\left(\kappa \backslash A_{n}\right)}, g\right]$.

Let $F_{n}=\left\{f_{\alpha}^{n}: \alpha<\alpha_{n}\right\}, \alpha_{n} \geqq \lambda$ exemplify this. Now define $f_{\alpha} \in{ }^{\kappa} \mu$ for $\alpha<\lambda$ as follows: for $\zeta<\kappa$ let $n(\zeta)=\operatorname{Min}\left\{n: \zeta \in A_{n}\right\}$ and $f_{\alpha}(\zeta)=\omega f_{\alpha}^{n(\zeta)}(\zeta)+n(\zeta)$. $\square_{6.1}$

6.3 Conclusion. Suppose $\operatorname{cf}(\kappa)>\aleph_{0}, \kappa>\sigma \geqq \aleph_{0}$ and $I=[\kappa]^{<\sigma}, \mu>\kappa^{\sigma}$. Then $T_{I}^{4}(\mu)$ is $T_{I}^{2}(\mu)$ hence is $T_{J}^{1}(\mu)$.

Proof. Apply 6.1 ( $\sigma^{+}$here corresponds to $\sigma$ there), more exactly by $6.1(\mathrm{~A})(2)$.

6.4 Remark. Asking on almost disjoint sets is an inessential change. 
REFERENCES.

[GHS] J. Gerlits, Andras Hajnal, and Z. Szentmiklossy. On the cardinality of certain Hausdorff spaces. Discrete Mathematics, 108:31-35, 1992. Topological, algebraical and combinatorial structures. Frolik's Memorial Volume.

[Sh:b] Saharon Shelah. Proper forcing, volume 940 of Lecture Notes in Mathematics. Springer-Verlag, Berlin-New York, xxix+496 pp, 1982.

[Sh 420] Saharon Shelah. Advances in Cardinal Arithmetic. In Finite and Infinite Combinatorics in Sets and Logic, pages 355-383. Kluwer Academic Publishers, 1993. N.W. Sauer et al (eds.).

[Sh 371] Saharon Shelah. Advanced: cofinalities of small reduced products. In Cardinal Arithmetic, volume 29 of Oxford Logic Guides, chapter VIII. Oxford University Press, 1994.

[Sh 355] Saharon Shelah. $\aleph_{\omega+1}$ has a Jonsson Algebra. In Cardinal Arithmetic, volume 29 of Oxford Logic Guides, chapter II. Oxford University Press, 1994.

[Sh 345a] Saharon Shelah. Basic: Cofinalities of small reduced products. In Cardinal Arithmetic, volume 29 of Oxford Logic Guides, chapter I. Oxford University Press, 1994.

[Sh 400] Saharon Shelah. Cardinal Arithmetic. In Cardinal Arithmetic, volume 29 of Oxford Logic Guides, chapter IX. Oxford University Press, 1994. Note: See also [Sh400a] below.

[Sh:g] Saharon Shelah. Cardinal Arithmetic, volume 29 of Oxford Logic Guides. Oxford University Press, 1994.

[Sh 345b] Saharon Shelah. Entangled Orders and Narrow Boolean Algebras. In Cardinal Arithmetic, volume 29 of Oxford Logic Guides. Oxford University Press, 1994. Appendix 2.

[Sh 430] Saharon Shelah. Further cardinal arithmetic. Israel Journal of Mathematics, 95:61-114, 1996. math.LO/9610226.

[Sh 462] Saharon Shelah. $\sigma$-entangled linear orders and narrowness of products of Boolean algebras. Fundamenta Mathematicae, 153:199-275, 1997. math.LO/9609216.

[Sh 666] Saharon Shelah. On what I do not understand (and have something to say:) Part I. Fundamenta Mathematicae, 166:1-82, 2000. math.LO/9906113.

[Sh 513] Saharon Shelah. PCF and infinite free subsets in an algebra. Archive for Mathematical Logic, 41:321-359, 2002. math.LO/9807177. 